\title{
Unitarization of the Horocyclic Radon Transform on Symmetric Spaces
}

Francesca Bartolucci, Filippo De Mari and Matteo Monti

Francesca Bartolucci

Seminar for Applied Mathematics, ETH Zurich, Raemistrasse 101, 8092 Zurich, Switzerland, email: francesca.bartolucci@sam.math.ethz.ch

Filippo De Mari

Department of Mathematics \& MaLGa Center, University of Genoa, Via Dodecaneso 35, 16146 Genova, Italy, e-mail: demari@dima.unige.it

Matteo Monti

Department of Mathematics \& MaLGa Center, University of Genoa, Via Dodecaneso 35, 16146 Genova, Italy, e-mail:m.monti@dima.unige.it 



\section{Contents}

Unitarization of the Horocyclic Radon Transform on Symmetric Spaces . 1 Francesca Bartolucci, Filippo De Mari and Matteo Monti

$1 \quad$ Introduction ................................... 3

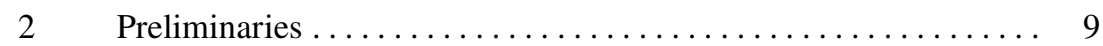

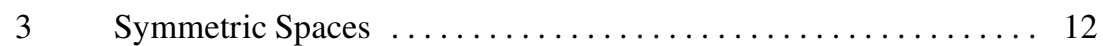

3.1 Riemannian Globally Symmetric Spaces . . . . . . . . . . 13

3.2 Types of Symmetric Spaces ................... 19

3.3 Boundary of a Symmetric Space ............... 21

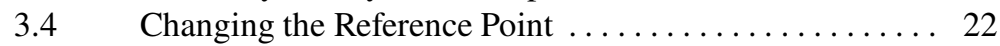

$3.5 \quad$ Horocycles ............................ 24

$4 \quad$ Analysis on Symmetric Spaces ....................... 32

$4.1 \quad$ Measures ............................ 33

4.2 The Helgason-Fourier Transform . ............. 41

4.3 The Horocyclic Radon Transform ................ 44

$5 \quad$ Unitarization and Intertwining $\ldots \ldots \ldots \ldots \ldots \ldots \ldots \ldots \ldots \ldots$

References ................................... 53

\section{Introduction}

The Radon transform has its origin in the problem of recovering a function defined on $\mathbb{R}^{d}$ from its integrals over hyperplanes. In 1917 Radon proved the reconstruction formula for two and three-dimensional signals. In $\mathbb{R}^{3}$ it reads

$$
f(x)=-\frac{1}{8 \pi^{2}} \Delta \int_{S^{2}} \mathcal{R} f(\theta, x \cdot \theta) \mathrm{d} \theta,
$$

where $\Delta$ is the Laplacian acting on the variable $x, S^{2}$ is the sphere in $\mathbb{R}^{3}$ and for every $\theta \in S^{2}$ and $t \in \mathbb{R}$ we denote with $\mathcal{R} f(\theta, t)$ the integral of $f$ over the hyperplane $x \cdot \theta=t$. Formula (1) suggests to define two dual transforms $f \mapsto \mathcal{R} f$, 
$g \mapsto \mathcal{R}^{\#} g$, known as Radon transform and dual Radon transform, or back-projection, respectively. The Radon transform $\mathcal{R}$ maps a function on $\mathbb{R}^{d}$ into the set of integrals over all hyperplanes, while the dual Radon transform $\mathcal{R}^{\#}$ maps a function defined on the set of hyperplanes of $\mathbb{R}^{d}$ into its integrals over the sheaves of hyperplanes through a point. Formula (1) can be rewritten

$$
f=-\frac{1}{2} \Delta \mathcal{R}^{\#} \mathcal{R} f
$$

and solves the inverse problem of recovering $f$ from the measured datum $\mathcal{R} f$.

This classical inverse problem is a particular case of the more general issue of recovering an unknown function on a manifold by means of its integrals over a family of submanifolds, already investigated by Gelfand in the 1950's [12]. A natural framework for such general inverse problems was considered by Helgason [17] and is motivated by the group structure hidden in the polar Radon transform setting [19], whereby the signals to be analyzed are in $\mathbb{R}^{d}$.

In the planar case, $\mathbb{R}^{2}$ and $[0,2 \pi) \times \mathbb{R}$, which parametrizes the set of lines in the plane by polar coordinates, are both transitive spaces of the rigid motions' group. This is $G=\mathbb{R}^{2} \rtimes K$, with $K=\left\{R_{\phi}: \phi \in[0,2 \pi)\right\}$ where

$$
R_{\phi}=\left[\begin{array}{cc}
\cos \phi & -\sin \phi \\
\sin \phi & \cos \phi
\end{array}\right] .
$$

We write $(b, \phi) \in \mathbb{R}^{2} \times[0,2 \pi)$ for the elements in $G$ and the group law is

$$
(b, \phi)\left(b^{\prime}, \phi^{\prime}\right)=\left(b+R_{\phi} b^{\prime}, \phi+\phi^{\prime} \bmod 2 \pi\right) .
$$

The group $G$ acts transitively on $\mathbb{R}^{2}$ by the action

$$
(b, \phi)[x]=R_{\phi} x+b
$$

and the isotropy at the origin $x_{0}=(0,0)$ is the Abelian subgroup

$$
K \simeq\{(0, \phi): \phi \in[0,2 \pi)\} .
$$

Therefore $\mathbb{R}^{2} \simeq G / K$ under the canonical isomorphism $g K \mapsto g\left[x_{0}\right]$. The group $G$ is a group of affine transformations of the plane and maps lines into lines. A line in the plane is parametrized by the direction ${ }^{t} n(\theta)=(\cos (\theta), \sin (\theta))$, where $\theta \in[0,2 \pi)$, of its normal and by the coordinate $t$ on the oriented normal line ${ }^{1}$ which describes its intersection with the given line. The action of $G$ is then given by

$$
(b, \phi) .(\theta, t)=\left(\theta+\phi \bmod 2 \pi, t+n(\theta) \cdot R_{\phi}^{-1} b\right)
$$

and is easily seen to be transitive. The isotropy at the $y$-axis $\xi_{0}=(0,0) \in[0,2 \pi) \times \mathbb{R}$ is

$$
H=\left\{\left(\left(0, b_{2}\right), \phi\right): b_{2} \in \mathbb{R}, \phi \in\{0, \pi\}\right\} .
$$

${ }^{1}$ The orientation is such that the coordinate $t$ is 1 exactly at $(\cos \theta, \sin \theta)$. 
Thus, $[0,2 \pi) \times \mathbb{R} \simeq G / H$ under the canonical isomorphism $g H \mapsto g . \xi_{0}$. From this group-theoretic point of view, the fact that a point $x \in \mathbb{R}^{2}$ belongs to the line $(\theta, t) \in$ $[0,2 \pi) \times \mathbb{R}$ is equivalent to requiring that the left cosets $x=g_{1} K$ and $(\theta, t)=g_{2} H$ intersect. Indeed, $g_{1}\left[x_{0}\right]$ belongs to the line $g_{2} . \xi_{0}$ if and only if there exists $h \in H$ such that $g_{1}\left[x_{0}\right]=g_{2} h\left[x_{0}\right]$, so that $g_{1}\left(g_{2} h\right)^{-1} \in K$ and $g_{1} K \cap g_{2} H \neq \emptyset$. This structure illustrates the following general framework introduced by Helgason.

Consider two $G$-spaces $X$ and $\Xi$, where the actions on $x \in X$ and $\xi \in \Xi$ are

$$
(g, x) \mapsto g[x], \quad(g, \xi) \mapsto g \cdot \xi .
$$

Both $X$ and $\Xi$ are assumed to be transitive spaces, so that there exist quasi-invariant measures $\mathrm{d} x$ and $\mathrm{d} \xi$. In Helgason's approach, it is assumed that $\mathrm{d} x$ and $\mathrm{d} \xi$ are invariant measures. Fix $x_{0} \in X$ and $\xi_{0} \in \Xi$ and denote by $K$ and $H$ the corresponding stability subgroups, so that $X \simeq G / K$ and $\Xi \simeq G / H$ under the isomorphisms $g K \mapsto g\left[x_{0}\right]$ and $g H \mapsto g \cdot \xi_{0}$, respectively. The space $X$ is meant to describe the ambient in which the functions to be analysed live, for example the Euclidean plane, or the sphere $S^{2}$ or the hyperbolic plane $H^{2}$. The second space $\Xi$ parametrises the set of submanifolds of $X$ over which one wants to integrate functions, for instance lines in the Euclidean plane, great circles in $S^{2}$, geodesics or horocycles in $H^{2}$. Motivated by the group structure behind the polar Radon transform, the elements in $\Xi$ can be realized as submanifolds of $X$ introducing the concept of incidence. Two elements $x=g_{1} K$ and $\xi=g_{2} H$ are said to be incident if they intersect as cosets in $G$. The concept of incidence translates the fact that a point $x \in X$ belongs to the submanifold parametrized by $\xi \in \Xi$. Any point $\xi \in \Xi$ is realized as a submanifold $\widehat{\xi} \subset X$ by taking all the points $x \in X$ that are incident to $\xi$. Precisely,

$$
\widehat{\xi}=\{x \in X: x \text { and } \xi \text { are incident }\} \subset X .
$$

Conversely, one builds the "sheaf" of manifolds $\check{x}$ through the point $x \in X$ by taking all the points $\xi \in \Xi$ that are incident to $x$

$$
\check{x}=\{\xi \in \Xi: \xi \text { and } x \text { are incident }\} \subset \Xi .
$$

By (2) and (3) we have that

$$
\widehat{\xi}_{0}=H\left[x_{0}\right] \subset X, \quad \check{x}_{0}=K . \xi_{0} \subset \Xi .
$$

Both $\check{x}_{0}$ and $\widehat{\xi}_{0}$ are transitive spaces and hence carry quasi-invariant measures. By definition, for any $x=g K$ and $\xi=\gamma H$

$$
\check{x}=g . \check{x}_{0} \subset \Xi, \quad \widehat{\xi}=\gamma\left[\widehat{\xi}_{0}\right] \subset X,
$$

which are closed subsets by Lemma 1.1 in [19]. If the maps $\xi \mapsto \widehat{\xi}$ and $x \mapsto \check{x}$ are both injective, then the pair of homogeneous spaces $(X, \Xi)$ is called a dual pair. This assumption is called transversality, see Lemma 1.3 in [19] for an equivalent characterization. The transversality condition avoids a redundant parametrisation of the submanifolds of $X$. The reader may consult [19] for numerous examples 
of dual pairs. It is worth observing that the leading example of the polar Radon transform does not satisfy the transversality condition. Indeed, the points $(\theta, t)$ and $(\theta+\pi \bmod 2 \pi,-t)$ in $[0,2 \pi) \times \mathbb{R}$ both parametrise the line given by the set of points

$$
\widehat{(\theta, t)}=(\theta+\pi \overline{\bmod 2} \pi,-t)=\left\{x \in \mathbb{R}^{2}: x \cdot n(\theta)=t\right\} .
$$

For a deeper study on the injectivity issue, the reader may consider [2].

In Helgason's approach the transitive spaces $\check{x}_{0}$ and $\widehat{\xi}_{0}$ are supposed to carry $K$-invariant and $H$-invariant measures, respectively, that is

$$
\begin{array}{cl}
\int_{\check{x}_{0}} g\left(k^{-1} \cdot \xi\right) \mathrm{d} \mu_{0}(\xi)=\int_{\check{x}_{0}} g(\xi) \mathrm{d} \mu_{0}(\xi), & g \in L^{1}\left(\check{x}_{0}, \mathrm{~d} \mu_{0}\right), k \in K, \\
\int_{\widehat{\xi}_{0}} f\left(h^{-1}[x]\right) \mathrm{d} m_{0}(x)=\int_{\widehat{\xi}_{0}} f(x) \mathrm{d} m_{0}(x), & g \in L^{1}\left(\widehat{\xi}_{0}, \mathrm{~d} m_{0}\right), h \in H .
\end{array}
$$

In order to define the Radon transform and its dual, one needs to introduce measures on $\widehat{\xi}$ and $\check{x}$. This may be done taking the pushforward of the measure $\mathrm{d} \mu_{0}$ to $\widehat{\xi}=(g H)^{\hat{\xi}}$ by the map $\widehat{\xi}_{0} \ni x \mapsto g[x] \in \widehat{\xi}$ and of the measure $\mathrm{d} m_{0}$ to $\check{x}=(g K)^{\nu}$ by the map $\check{x}_{0} \ni \xi \mapsto g . \xi \in \check{x}$, respectively. We denote by $\mathrm{d} \mu_{x}$ the measure on $\check{x}$ and by $\mathrm{d} m_{\xi}$ the measure on $\widehat{\xi}$. Since the measures on $\widehat{\xi}_{0}$ and $\check{x}_{0}$ are invariant, the measures $\mathrm{d} m_{\xi}$ and $\mathrm{d} \mu_{x}$ do not depend on the choice of the representatives of $\xi$ and $x$ and the transversality condition guarantees that they are unique.

Definition 1. The Radon transform of $f$ is the map $\mathcal{R} f: \Xi \rightarrow \mathbb{C}$ given by

$$
\mathcal{R} f(\xi)=\int_{\widehat{\xi}} f(x) \mathrm{d} m_{\xi}(x),
$$

and the dual Radon transform of $g$ is the map $\mathcal{R}^{\#} g: X \rightarrow \mathbb{C}$ given by

$$
\mathcal{R}^{\#} g(x)=\int_{\check{x}} g(\xi) \mathrm{d} \mu_{x}(\xi),
$$

for any $f$ and $g$ for which the integrals converge.

Observe that, even if the transversality condition is not satisfied for the polar Radon transform, both $\widehat{(\theta, t)}$ and $(\theta+\pi \overline{\bmod 2} \pi,-t)$ are endowed with the same measure since the arc-length measure is invariant under translations and rotations. For this reason the polar Radon transform satisfies

$$
\mathcal{R}^{\mathrm{pol}} f(\theta, t)=\mathcal{R}^{\mathrm{pol}} f(\theta+\pi \bmod 2 \pi,-t) .
$$

In this context, the most relevant issue is to recover $f$ from the values of $\mathcal{R} f$. Another central issue is to prove that the Radon transform, up to a composition with a suitable pseudo-differential operator, can be extended to a unitary map $Q$ from $L^{2}(X, \mathrm{~d} x)$ to $L^{2}(\Xi, \mathrm{d} \xi)$ intertwining the quasi-regular representations $\pi$ and $\hat{\pi}$ of $G$ acting on $L^{2}(X, \mathrm{~d} x)$ and $L^{2}(\Xi, \mathrm{d} \xi)$, respectively. 
In [4], the authors obtain both an intertwining and a unitarization result for the affine Radon transform. The techniques used in [4] mimic the approach followed by Helgason to unitarize the polar Radon transform [19].

Later, inspired by the results in [4] a new approach based on representation theory has been taken in order to treat in a general and unified way the problem of unitarizing and inverting the Radon transform [1] under the assumption that $\pi$ and $\hat{\pi}$ are irreducible. The approach taken in [1], [4] differs from Helgason's since the assumptions on the measures carried by $X$ and $\Xi$ and by the submanifolds $\hat{\xi} \subset X$ are weaker, namely their relative invariance instead of (proper) invariance. This allows to consider a wider variety of cases of interest in applications, such as the similitude group studied by Murenzi [3], and the generalized shearlet dilation groups introduced by Führ in [9], [10] for the purpose of generalizing the standard shearlet group introduced in [23], [6]. It is assumed that there exists a non-trivial $\pi$-invariant subspace $\mathcal{A}$ of $L^{2}(X, \mathrm{~d} x)$ such that $\mathcal{R}$ is well defined for all $f \in \mathcal{A}$ and the adjoint of the operator $\mathcal{R}: \mathcal{A} \rightarrow L^{2}(\Xi, \mathrm{d} \xi)$ has non-trivial domain. Then, it is proved that the Radon transform $\mathcal{R}$ is a closable operator from $\mathcal{A}$ into $L^{2}(\Xi, \mathrm{d} \xi)$ and that its closure $\overline{\mathcal{R}}$ is independent of the choice of $\mathcal{A}$ and is the unique closed extension of $\mathcal{R}$. The main result states that if the quasi regular representations $\pi$ of $G$ on $L^{2}(X, \mathrm{~d} x)$ and $\hat{\pi}$ of $G$ on $L^{2}(\Xi, \mathrm{d} \xi)$ are irreducible, then the Radon transform $\mathcal{R}$, up to a composition with a suitable pseudo-differential operator, can be extended to a unitary operator $Q: L^{2}(X, \mathrm{~d} x) \rightarrow L^{2}(\Xi, \mathrm{d} \xi)$ which intertwines them, namely

$$
\hat{\pi}(g) Q \pi(g)^{-1}=Q, \quad g \in G .
$$

The proof is based on the extension of Schur's lemma due to Duflo and Moore [7].

A direct consequence of the result above is studied in [1]. Adding the hypothesis of square-integrability of $\pi$, the authors derive a new general inversion formula for the Radon transform of the form

$$
f=\int_{G} \chi(g)\langle\mathcal{R} f, \hat{\pi}(g) \Psi\rangle \pi(g) \psi \mathrm{d} g,
$$

where $\chi$ is a character of $G$ and $\psi \in L^{2}(X, \mathrm{~d} x)$ and $\Psi \in L^{2}(\Xi, \mathrm{d} \xi)$ are suitable mother wavelets and where the Haar integral is weakly convergent. Such formula is obtained by the usual reconstruction formula for square-integrable representations and then by applying the unitary operator $Q$ to both entries of the scalar product $\langle f, \pi(g) \psi\rangle$. We stress that the above formula allows to reconstruct an unknown signal by computing the family of coefficients $\{\langle\mathcal{R} f, \hat{\pi}(g) \Psi\rangle\}_{g \in G}$.

The results achieved in [1] and [4] have posed many interesting mathematical challenges. A natural question is to investigate how to generalize these findings to other groups and related representations without the hypothesis of irreducibility, because the techniques used in [1] cannot be transferred directly.

In this direction, we have considered in [5] the case of homogeneous trees. Precisely, we construct the unitarization of the horocyclic Radon transform on a homogeneous tree $X$ and we prove that it intertwines the quasi regular representations of the group of isometries of $X$ acting on the space of square-integrable functions 
on the tree itself and on the space of horocycles, respectively. Since the quasi regular representation is not irreducible, we adopt a combination of the approach followed by Helgason in the context of symmetric spaces [17] and the techniques that have been developed in [4]. The main observation motivating [5] is that homogeneous trees are the natural discrete counterpart of rank-one symmetric spaces.

This article is devoted to investigate the unitarization problem in the case when $X$ is a symmetric space and $\Xi$ is the set of horocycles of $X$, which has at large been addressed by Helgason. A remarkable difference from the cases treated in [1] is that the quasi regular representations $\pi$ of the group of isometries of the symmetric space $X$ acting on $L^{2}(X)$ is not irreducible, nor is it the representation $\hat{\pi}$ on $L^{2}(\Xi)$. We are well aware that the unitarization problem was already addressed and essentially solved by Helgason in [17]. Precisely, he constructs a pseudo-differential operator $\Lambda$ and he proves that the pre-composition with the horocyclic Radon transform yields an isometric operator, see Theorem 3.9 in Chap. II in [17]. Here, we prove that the composition $\Lambda \mathcal{R}$ can actually be extended to a unitary operator $Q: L^{2}(X, \mathrm{~d} x) \rightarrow$ $L_{b}^{2}(\Xi, \mathrm{d} \xi)$, where $\mathrm{d} x$ and $\mathrm{d} \xi$ are the $G$-invariant measures and where $L_{b}^{2}(\Xi, \mathrm{d} \xi)$ is a closed subspace of $L^{2}(\Xi, \mathrm{d} \xi)$ which accounts for the Weyl symmetries. Furthermore, we are able to show that $Q$ intertwines the quasi regular representations $\pi$ and $\hat{\pi}$.

This work is focused on the horocyclic Radon transform, but another interesting setting could be obtained by considering geodesics. Such Radon transform is commonly called X-ray transform and has been introduced and inverted by Helgason on the hyperbolic space $\mathbb{H}^{n}$, see Theorem 3.12 in Chap.I in [17], and on symmetric spaces of the noncompact type by Rouvière [24]. Although it is not in general true that a horocycle has codimension one in the symmetric space, the horocyclic Radon transform can be seen as the analogue of the Euclidean Radon transform on hyperplanes in $\mathbb{R}^{n}$, whereas the X-ray transform is the analogue of the Radon on lines in $\mathbb{R}^{n}$.

The primary reason of the present contribution was to settle the unitarization issue in the setup of noncompact symmetric spaces in all details, in a self-contained and accessible way to the readers that have little experience with the heavy machinery of semisimple groups. We do make use of the basic Lie theoretic notions but avoid as much as possible to make extensive use of the full body of the theory. Rather, we collect all the most relevant results of the theory that may serve as a map.

We are not aware of a general statement such as our Theorem 11 in the literature, though it is quite clear to us that the result comes as no surprise if not for the flexibility of our proof (see once again [4], [5]). We also believe that the material presented here is a readable introduction to a subject that may attract the attention of a wide community of young researchers.

The chapter is organized as it follows. In Sect. 2 we recall the basic facts of the analysis on semisimple Lie groups and we introduce the notation used throughout in the geometric analysis on noncompact Riemannian symmetric spaces. In Sect. 3 we present a brief overview of the general theory of symmetric spaces enriched with the examples of the Euclidean space, the sphere, the upper half plane, the unit disk and the positive definite symmetric matrices. Of particular interest for our purposes are $\S 3.3,3.4$ and 3.5 . In $\S 3.3$ we present the notion of boundary of a symmetric space and 
in $\S 3.4$ we show the infinitely many ways to represent it changing the reference point in the symmetric space. Finally, in $\S 3.5$ we define the family of horocycles and we prove some technical results needed in Sects. 4 and 5. In Sect. 4 we collect the analytic ingredients that come into play. We endow the symmetric space, its boundary and the family of horocycles with invariant measures. We introduce the Helgason-Fourier transform and its main features. Then, we study the horocyclic Radon transform and we discuss its relation with the Helgason-Fourier transform. Finally, in Sect. 5 we prove the unitarization result for the horocyclic Radon transform.

\section{Preliminaries}

The purpose of the introductory section is to recall the basic facts of the analysis on semisimple Lie groups and to establish the notation used throughout in the geometric analysis on noncompact Riemannian symmetric spaces. For a concise and effective exposition, see [16]. Classical references with a wider scope are [15], [17] and [22]. For a detailed introduction to differential geometry and Lie groups, we refer to [27].

A Lie algebra $\mathfrak{g}$ is simple if it is not Abelian and contains no proper Abelian ideals. A semisimple Lie algebra is then the Lie algebra direct sum of (all) its simple ideals. Cartan proved that on every semisimple Lie algebra $\mathfrak{g}$ there exists a Cartan involution $\theta$, namely an involution such that the symmetric bilinear form $B_{\theta}(X, Y)=-B(X, \theta Y)$ is positive definite, where $B$ is the usual Killing form defined by $B(X, Y)=\operatorname{tr}(\operatorname{ad} X \circ \operatorname{ad} Y)$. Such an involution gives rise to a Cartan decomposition of the Lie algebra, namely a vector space direct sum $\mathfrak{g}=\mathfrak{f}+\mathfrak{p}$, where $\mathfrak{f}$ and $\mathfrak{p}$ are the +1 and -1 eigenspaces of $\mathfrak{g}$ relative to $\theta$, respectively.

Fix a maximal Abelian subspace $\mathfrak{a}$ of $\mathfrak{p}$. The set $\{\operatorname{ad} H: H \in \mathfrak{a}\}$ is a commuting family of self-adjoint linear maps. Therefore, $\mathfrak{g}$ is the $B_{\theta}$-orthogonal direct sum of their joint eigenspaces, all of the eigenvalues of which are real and depend linearly on $H$. For any fixed $\alpha \in \mathfrak{a}^{*}$, the linear dual of $\mathfrak{a}$, we write

$$
\mathfrak{g}_{\alpha}=\{X \in \mathfrak{g}:(\operatorname{ad} H) X=\alpha(H) X \text { for all } H \in \mathfrak{a}\}
$$

and we say that $\alpha \neq 0$ is a restricted root, or simply a root of the pair $(\mathfrak{g}, \mathfrak{a})$, whenever $\mathfrak{g}_{\alpha} \neq\{0\}$. The set of restricted roots is $\Sigma$ and the spaces $\mathfrak{g}_{\alpha}$ with $\alpha \in \Sigma$ are called (restricted) root spaces.

An element $H \in \mathfrak{a}$ is called regular if $\alpha(H) \neq 0$ for all $\alpha \in \Sigma$, otherwise it is singular. The set $\mathfrak{a}^{\prime}$ of regular elements is the complement in $\mathfrak{a}$ of finitely many hyperplanes and its connected components are called the Weyl chambers.

We fix a Weyl chamber $\mathfrak{a}^{+} \subset \mathfrak{a}$ and we declare a root $\alpha$ to be positive if it has positive values on $\mathfrak{a}^{+}$. A root is simple if it cannot be written as the sum of positive roots. The set $\Delta$ of simple roots turns out to be a basis of $\mathfrak{a}^{*}$. Thus, there are exactly $\ell=\operatorname{dim} \mathfrak{a}$ simple roots. This number is an important invariant and is called the real rank of $\mathfrak{g}$. We order the elements in $\mathfrak{a}^{*}$, hence the roots in $\Sigma$, lexicographically with 
respect to an ordering $\delta_{1}, \ldots, \delta_{\ell}$ of the simple roots. This means that $\lambda=\sum a_{j} \delta_{j}$ is positive (written $\lambda>0$ ) if the first non-zero coefficient $a_{k}$ is positive. Together with $\mathfrak{g}, \theta$ and $\mathfrak{a}$ we assume that an ordering " $>$ " has been fixed on $\mathfrak{a}^{*}$ by choosing a labeling of the simple roots relative to a fixed Weyl chamber $\mathfrak{a}^{+}$. We consequently denote by $\Sigma^{+}$and $\Sigma^{-}$the positive and negative roots, respectively. Clearly, $\Sigma=\Sigma^{+} \cup \Sigma^{-}$, a disjoint union.

If $G$ is a Lie group, then it is said to be semisimple if such is its Lie algebra. Furthermore, for any Cartan involution $\theta$ on its Lie algebra $\mathfrak{g}$ there exists an automorphism $\Theta$ of $G$ such that $d \Theta=\theta$ and $\Theta^{2}=$ id.

Theorem 1 (The Iwasawa decomposition). Let $G$ be a connected semisimple Lie group, $\mathfrak{g}=\mathfrak{f}+\mathfrak{p}$ be a Cartan decomposition of its Lie algebra and fix a maximal Abelian subspace $\mathfrak{a}$ of $\mathfrak{p}$ and an ordering on $\mathfrak{a}^{*}$. The vector space direct sum

$$
\mathfrak{n}=\sum_{\alpha \in \Sigma^{+}} \mathfrak{g}_{\alpha}
$$

is a nilpotent Lie algebra and $\mathfrak{g}$ decomposes as the vector space direct sum

$$
\mathfrak{g}=\mathfrak{f}+\mathfrak{a}+\mathfrak{n} .
$$

Furthermore, let $K, A$ and $N$ be the connected subgroups of $G$ whose Lie algebras are $\mathfrak{f}, \mathfrak{a}$ and $\mathfrak{n}$, respectively. The multiplication map $K \times A \times N \rightarrow G$ given by $(k, a, n) \mapsto$ kan is a diffeomorphism. The groups $A$ and $N$ are simply connected and $A N$ is solvable.

Observe that $A N$ is in fact a semidirect product. Indeed, $A$ acts on $N$ by conjugation, as is most rapidly seen by observing that $\operatorname{Ad} a(X) \in \mathfrak{g}_{\alpha}$ if $X \in \mathfrak{g}_{\alpha}$ for any root $\alpha \in \Sigma$ and for all $a \in A$. Indeed, for any $H \in \mathfrak{a}$, since $\mathfrak{a}$ is Abelian, one has

$$
[H, \operatorname{Ad} a(X)]=\operatorname{Ad} a\left(\left[\operatorname{Ad} a^{-1}(H), X\right]\right)=\operatorname{Ad} a([H, X])=\alpha(H) \operatorname{Ad} a(X) .
$$

Therefore $\operatorname{Ad} a$ preserves root spaces and in particular it preserves $\mathfrak{n}$. Thus $A$ acts on $\mathfrak{n}$ via the adjoint action and, passing to exponentials, it acts on $N$ by conjugation. This is tantamount to saying that $A$ normalizes $N$ inside $G$. Hence $N A=A N$ is the semidirect product $N \rtimes A$.

Let $M$ and $M^{\prime}$ denote the centralizer and normalizer of $\mathfrak{a}$ in $K$, respectively. This means that

$$
\begin{aligned}
M & =\{m \in K: \operatorname{Ad} m(H)=H \text { for all } H \in \mathfrak{a}\} \\
M^{\prime} & =\{w \in K: \operatorname{Ad} w(H) \in \mathfrak{a} \text { for all } H \in \mathfrak{a}\} .
\end{aligned}
$$

Passing to exponentials, it follows that if $m \in M$, then $\mathrm{mam}^{-1}=a$ for all $a \in A$ and if $w \in M^{\prime}$, then $w a w^{-1} \in A$ for all $a \in A$. The quotient group $W=M^{\prime} / M$ is called the Weyl group of $(G, K)$. The compact Lie groups $M$ and $M^{\prime}$ have the same Lie algebra, namely $\mathrm{m}$, so that $W$ is in fact a finite group. The Weyl group $W$ acts on $\Sigma$ 
by

$$
(w \cdot \alpha)(H)=\alpha\left(\operatorname{Ad} w^{-1} H\right), \quad H \in \mathfrak{a} .
$$

The very same formula defines an action on the whole dual space $\mathfrak{a}^{*}$. It is worth observing that the action of $W$ on $\mathfrak{a}^{*}$ maps Weyl chambers in Weyl chambers in a free and transitive way (see ...). So that, the cardinality of the Weyl chambers coincides with $|W|$. For any $\alpha \in \Sigma$, the vector space dimension of $\mathfrak{g}_{\alpha}$ is called the multiplicity of $\alpha$ and is usually denoted $m_{\alpha}$. The following element of $\mathfrak{a}^{*}$ plays a crucial role in the theory:

$$
\rho=\frac{1}{2} \sum_{\alpha \in \Sigma^{+}} m_{\alpha} \alpha
$$

This linear functional on $\mathfrak{a}$ naturally appears in relation with the semidirect product structure of the Iwasawa group $A N$, see (29).

Example: the decomposition of $\operatorname{SL}(d, \mathbb{R})$. We consider the Lie algebra $\mathfrak{g}=$ $\mathfrak{s l}(d, \mathbb{R})$ of $G=\operatorname{SL}(d, \mathbb{R})$, namely

$$
\mathfrak{s l}(d, \mathbb{R})=\{X \in \mathfrak{g l}(d, \mathbb{R}): \operatorname{tr} X=0\} .
$$

The Cartan decomposition associated to the standard involution $\theta(X)=-{ }^{t} X$ reads

$$
\mathfrak{s l}(d, \mathbb{R})=\mathfrak{s v}(d, \mathbb{R})+\operatorname{Sym}_{0}(d),
$$

where $\mathfrak{p}=\operatorname{Sym}_{0}(d)$ is the space of $d \times d$ symmetric and traceless real matrices. The Cartan involution $\Theta$ for $\operatorname{SL}(d, \mathbb{R})$ is then

$$
\Theta g={ }^{t} g^{-1}
$$

as for all matrix groups with real entries. Hence $K=\mathrm{SO}(d)$, a maximal compact subgroup of $\operatorname{SL}(d, \mathbb{R})$. The diffeomorphism $(k, X) \mapsto k \exp X$ of $\operatorname{SO}(d) \times \operatorname{Sym}_{0}(d) \rightarrow G$ is just the classical polar decomposition. The center of $\operatorname{SL}(d, \mathbb{R})$ is the identity matrix if $d$ is odd and $\{ \pm \mathrm{id}\}$ if $d$ is even. The natural maximal Abelian subspace of $\operatorname{Sym}_{0}(d)$ is the $(d-1)$-dimensional vector space consisting of the diagonal matrices $\operatorname{diag}\left(a_{1}, \ldots, a_{d}\right)$ with $a_{1}+\cdots+a_{d}=0$. Thus, the real rank of $\mathfrak{s l}(d, \mathbb{R})$ is $d-1$. Let $E_{i j}$ denote the matrix whose only non-zero entry is 1 at position $(i, j)$. Then, for $H=\operatorname{diag}\left(a_{1}, \ldots, a_{d}\right)$ and $i \neq j$

$$
\left[H, E_{i j}\right]=\left(a_{i}-a_{j}\right) E_{i j}
$$

and in fact $E_{i j}$ spans a root space provided that $i \neq j$. It is customary to introduce the linear functionals $e_{k}(\cdot)$ on $\mathfrak{a}$, with $1 \leq k \leq d$, via $e_{k}\left(\operatorname{diag}\left(a_{1}, \ldots, a_{d}\right)\right)=a_{k}$. Thus, for $i \neq j$ the (restricted) root $\alpha_{i j}=e_{i}-e_{j}$ acts on $H=\operatorname{diag}\left(a_{1}, \ldots, a_{d}\right)$ by

$$
\alpha_{i j}(H)=a_{i}-a_{j}
$$

and we write in simplified form $\mathfrak{g}_{i j}$ in place of $\mathfrak{g}_{\alpha_{i j}}$ for the root space 


$$
\mathfrak{g}_{i j}=\operatorname{sp}\left\{E_{i j}\right\}, \quad i \neq j .
$$

For $i<j$ the matrix $E_{i j}$ is upper triangular, and for $i>j$ it is lower triangular. A natural choice of Weyl chamber is

$$
\mathfrak{a}^{+}=\left\{\operatorname{diag}\left(a_{1}, \ldots, a_{d}\right): a_{1}>a_{2}>\cdots>a_{d}\right\} .
$$

It is immediate to check that for $j=1, \ldots, d-1$ the roots $\delta_{j}=e_{j}-e_{j+1}$ are the simple ones and that the set of positive roots is

$$
\Sigma^{+}=\left\{\alpha_{i j}: i<j\right\}
$$

It follows that the nilpotent Iwasawa Lie algebra $\mathfrak{n}$ defined in (4) is just the Lie algebra of strictly upper triangular matrices. Notice that $\mathfrak{g}_{0}=\mathfrak{a}$, that is, $\mathfrak{m}=\{0\}$ and that $\operatorname{dim} \mathfrak{g}_{\alpha}=1$ for every restricted $\operatorname{root} \alpha \in \Sigma$. Hence the functional $\rho$ has the form

$$
\rho(H)=\frac{1}{2} \sum_{i<j} \alpha_{i j}(H)=\frac{1}{2} \sum_{i<j}\left(a_{i}-a_{j}\right)=\sum_{j=1}^{d}\left(\frac{d+1}{2}-j\right) a_{j} .
$$

Let $A$ be the group of diagonal matrices with positive entries and determinant 1 , namely

$$
\operatorname{diag}\left(\mathrm{e}^{a_{1}}, \ldots, \mathrm{e}^{a_{d}}\right), \quad a_{1}+\cdots+a_{d}=0,
$$

and let $N$ be the group of unipotent upper triangular matrices, namely those of the form

$$
\left[\begin{array}{ccccc}
1 & a_{12} & \ldots & \ldots & a_{1, d} \\
0 & 1 & \ddots & & \vdots \\
\vdots & \ddots & \ddots & \ddots & \vdots \\
\vdots & & \ddots & 1 & a_{d-1, d} \\
0 & \ldots & \ldots & 0 & 1
\end{array}\right] .
$$

Then $\mathfrak{a}$ and $\mathfrak{n}$ are the Lie algebra of $A$ and $N$, respectively. Hence $\operatorname{SL}(d, \mathbb{R}) \simeq K A N$ by the Iwasawa decomposition.

\section{Symmetric Spaces}

Symmetric spaces are very special kinds of homogeneous spaces. The reader is assumed to be familiar with basic Differential Geometry and in particular with the main results on group actions and homogeneous spaces. The natural reference for the material in this section is the celebrated monography [15] by Helgason, of which this is a synthesis with examples. Other sources are for example [21], [28].

We very briefly recall the basic facts that we shall use throughout. A homogeneous space $X$ is a transitive $G$-space. Saying that $X$ is a $G$-space means that we are given 
a continuous map $G \times X \rightarrow X$, written $(g, x) \mapsto g x$ and called an action of $G$ on $X$, which satisfies

(i) $x \mapsto g x$ is a homeomorphism of $X$ for each $g \in G$,

(ii) $g(h x)=(g h) x$ for all $g, h \in G$ and $x \in X$.

The $G$-space $X$ is called transitive if for every $x, y \in X$ there exists $g \in G$ such that $g x=y$. In this case $X$ is indentified with $G / H$ through the action of $G$, where $H$ is the isotropy subgroup at some point $x_{0} \in X$, namely

$$
H=\left\{g \in G: g x_{0}=x_{0}\right\} .
$$

This identification depends on the choice of the reference point $x_{0} \in X$ and is given by the bijection

$$
G / H \rightarrow X, \quad g H \mapsto g x_{0} .
$$

If we choose a different reference point $x_{0}^{\prime}=g_{0} x_{0}$ for some $g_{0} \in G$, it is sufficient to replace $H$ with $H^{\prime}=g_{0} H g_{0}^{-1}$. The map $g \mapsto g_{0} g g_{0}^{-1}$ induces a $G$-equivariant homeomorphism between $G / H$ and $G / H^{\prime}$. If the topology on $G / H$ is the quotient topology then the identification map is actually a homeomorphism.

In the present contribution, we often consider different $G$-spaces of the same group. For clarity, we shall thus adopt notational variations to distinguish among different actions, such as $g[x]$ or $g . x$ or $g \cdot x$ or $g\langle x\rangle$ and so forth.

\subsection{Riemannian Globally Symmetric Spaces}

Let $\mathcal{M}$ be a Riemannian manifold and let $I(\mathcal{M})$ denote the group of isometries of $\mathcal{M}$. We shall endow $I(\mathcal{M})$ with the compact-open topology, the smallest topology in which all the sets

$$
W(C, U)=\{g \in I(\mathcal{M}): g(C) \subset U\}
$$

are open, where $C$ varies in the compacta of $\mathcal{M}$ and $U$ in the open sets.

Theorem 2 (Theorem 2.5, Chap. IV,[15]). Let $\mathcal{M}$ be a Riemannian manifold.

(i) The group of isometries $I(\mathcal{M})$ with the compact-open topology is a locally compact topological group acting on $\mathcal{M}$.

(ii) The isotropy subgroup of $I(\mathcal{M})$ at any point of $\mathcal{M}$ is compact.

Definition 2. The Riemannian manifold $\mathcal{M}$ is a Riemannian globally symmetric space if each $p \in \mathcal{M}$ is an isolated fixed point of an isometry $\sigma_{p}$ of $\mathcal{M}$ that is involutive $\left(\sigma_{p}^{2}=\mathrm{id}\right)$.

It may be shown that each $\sigma_{p}$ is in this case unique and that there exists a neighborhood $N_{p}$ of $p$ in which $\sigma_{p}$ is the geodesic symmetry. This means that if $q \in N_{p}$ and $\gamma(t)$ is the geodesic such that $\gamma(0)=p$ and $\gamma(1)=q$, then $\sigma_{p}(q)=\gamma(-1)$. 
Euclidean space. Let $\mathcal{M}=\mathbb{R}^{n}$ and fix $p \in \mathbb{R}^{n}$. The globally defined map $\sigma_{p}(x)=$ $2 p-x$ is clearly involutive and isometric with respect to the the Euclidean distance because $\left\|\sigma_{p}(x)-\sigma_{p}(y)\right\|=\|y-x\|$. Further, $\sigma_{p}(x)=x$ if and only if $x=p$, so $p$ is an isolated fixed point.

The sphere. Let $\mathcal{M}=S^{n-1}$ and consider the map defined on $\mathbb{R}^{n}$ by $x \mapsto \Omega x$ where

$$
\Omega=\left[\begin{array}{ll}
1 & \\
& -\mathrm{I}_{n}
\end{array}\right] \text {. }
$$

Evidently, it leaves the unit sphere invariant and is an isometry with respect to the natural Riemannian structure on it. It fixes the north pole $e_{0}=(1,0, \ldots, 0)$. Next choose $p \in \mathcal{M}$ and take $R \in \mathrm{SO}(n)$ such that $p=R e_{0}$. Then $\sigma_{p}=R \Omega R^{-1}$ is the required involutive isometry, as the reader is invited to check.

The upper half plane. Let $\mathcal{M}$ denote the upper half plane, which we think of as one of the natural models of the 2-dimensional hyperbolic space. We realize it as the complex numbers with positive imaginary part. The Riemannian structure on $\mathcal{M}$ is given by the inner product

$$
\langle u, v\rangle_{z}=\frac{(u, v)}{4 y^{2}}
$$

where $u, v \in T_{z}(\mathcal{M})$ are tangent vectors at $z=x+i y \in \mathcal{M}$. It is important to observe that $G=\operatorname{SL}(2, \mathbb{R})$ acts transitively on $\mathcal{M}$ by means of the Möbius action, namely

$$
g[z]=\left[\begin{array}{ll}
a & b \\
c & d
\end{array}\right][z]=\frac{a z+b}{c z+d} .
$$

The imaginary part of $g[z]$ is positive if such is that of $z$, so that (7) is indeed an action. To show transitivity, we fix $p=b+i a \in \mathcal{M}$ with $a>0$ and consider

$$
g_{p}=\left[\begin{array}{ll}
1 & b \\
0 & 1
\end{array}\right]\left[\begin{array}{cc}
\sqrt{a} & 0 \\
0 & 1 / \sqrt{a}
\end{array}\right]=\left[\begin{array}{cc}
\sqrt{a} & b / \sqrt{a} \\
0 & 1 / \sqrt{a}
\end{array}\right],
$$

an element of the Iwasawa subgroup $N A$ of $\operatorname{SL}(2, \mathbb{R})$. It is immediate to check that $g_{p}[i]=p$ and that the isotropy group at $i$ is $K=\operatorname{SO}(2)$, so that $\mathcal{M} \simeq G / K$.

As for the isometric involutions, consider first the Möbius action induced by

$$
J=\left[\begin{array}{cc}
0 & 1 \\
-1 & 0
\end{array}\right]
$$

which is the map $z \mapsto-1 / z$, namely $x+i y \mapsto(-x+i y) /\left(x^{2}+y^{2}\right)$, and may also be described in polar coordinates by

$$
\rho(\cos \theta+i \sin \theta) \mapsto \frac{1}{\rho^{2}}(-\cos \theta+i \sin \theta) .
$$


This fixes only $i$ (for $\rho=1$ and $\theta=\pi / 2$ ) and is thus a global involution of which $i$ is an isolated fixed point. A global involution fixing only the point $p$ is given by the Möbius action of the $\operatorname{SL}(2, \mathbb{R})$ element $g_{p} J g_{p}^{-1}$.

Of course, it needs to be to seen that these maps are indeed isometries relative to the hyperbolic distance. To this end, observe that any differentiable path $\gamma:[a, b] \rightarrow \mathcal{M}$, with $\gamma(t)=x(t)+i y(t)$ has length

$$
L(\gamma)=\int_{a}^{b}\langle\dot{\gamma}(t), \dot{\gamma}(t)\rangle^{1 / 2} \mathrm{~d} t=\frac{1}{2} \int_{a}^{b} \frac{\sqrt{\dot{x}^{2}(t)+\dot{y}^{2}(t)}}{y(t)} \mathrm{d} t .
$$

It is then very easy to check that $L(g[\gamma])=L(\gamma)$ if $g$ is either $J$ or any of the following

$$
\left[\begin{array}{cc}
\mathrm{e}^{s} & 0 \\
0 & \mathrm{e}^{-s}
\end{array}\right] \in A, \quad\left[\begin{array}{ll}
1 & t \\
0 & 1
\end{array}\right] \in N
$$

We now show that these are enough. Indeed, any lower triangular unipotent matrix in $G$ is of the form $J n J^{-1}$ for some $n \in N$. Next, any rotation in $\mathrm{SO}(2)$ with $\cos \theta \neq 0$ can be written

$$
\left[\begin{array}{cc}
\cos \theta & \sin \theta \\
-\sin \theta & \cos \theta
\end{array}\right]=\left[\begin{array}{cc}
1 & \tan \theta \\
0 & 1
\end{array}\right]\left[\begin{array}{cc}
1 / \cos \theta & 0 \\
0 & \cos \theta
\end{array}\right]\left[\begin{array}{cc}
1 & 0 \\
-\tan \theta & 1
\end{array}\right] .
$$

The rotations with $\cos \theta=0$ are of the form $\pm J$, and we conclude that any element in $K$ is a finite product of elements ${ }^{2}$ chosen in $\{ \pm J\} \cup A \cup N$. By the Iwasawa decomposition we conlcude that in fact $L(g[\gamma])=L(\gamma)$ for any $g \in G$. This entails that $\operatorname{SL}(2, \mathbb{R})$ acts by isometries on $\mathcal{M}$. It is worth mentioning that the isometry group of the upper half plane is generated by $\operatorname{SL}(2, \mathbb{R})$ and by the map $z \mapsto 1 / \bar{z}$.

The unit disk. A second natural model of the 2-dimensional hyperbolic space is the unit disk $\mathcal{M}=\{z \in \mathbb{C}:|z|<1\}$, later denoted $\mathbb{D}$. This is the Riemannian manifold with inner product

$$
\langle u, v\rangle_{z}=\frac{(u, v)}{\left(1-|z|^{2}\right)^{2}}
$$

where $u, v \in T_{z}(\mathcal{M})$ are tangent vectors at $z \in \mathcal{M}$. The group

$$
G=\mathrm{SU}(1,1):=\left\{\left[\begin{array}{ll}
a & b \\
\bar{b} & \bar{a}
\end{array}\right]: a, b \in \mathbb{C},|a|^{2}-|b|^{2}=1\right\}
$$

acts on $\mathcal{M}$ by the very same Möbius action as given by (7). Following similar reasoning as above, we can prove that the action is transitive using the $N A$ action on the point $0 \in \mathcal{M}$, where the Iwasawa components of $G$ are obtained from that of $\operatorname{SL}(2, \mathbb{R})$ by conjugating within $\operatorname{SL}(2, \mathbb{C})$ first with a $\pi / 4$-rotation and then with $\Lambda^{-1}$, where

$$
\Lambda=\frac{1}{\sqrt{2}}\left[\begin{array}{ll}
1 & i \\
i & 1
\end{array}\right]
$$

\footnotetext{
2 This argument is nothing else but the Bruhat decomposition of $\operatorname{SL}(2, \mathbb{R})$.
} 
The Iwasawa subgroups are explicitly given by

$$
\begin{aligned}
& K=\left\{\left[\begin{array}{cc}
\mathrm{e}^{i \theta} & 0 \\
0 & \mathrm{e}^{-i \theta}
\end{array}\right]: \theta \in[0,2 \pi)\right\}, \\
& A=\left\{\left[\begin{array}{cc}
\cosh t & \sinh t \\
\sinh t & \cosh t
\end{array}\right]: t \in \mathbb{R}\right\}, \\
& N=\left\{\left[\begin{array}{cc}
1+i s & -i s \\
i s & 1-i s
\end{array}\right]: s \in \mathbb{R}\right\} .
\end{aligned}
$$

Of course the isotropy at $o \in \mathcal{M}$ is $K$ and $\mathcal{M} \simeq G / K$. The reader is invited to write the isometric involutions that prove $\mathcal{M}$ to be a symmetric space. We content ourselves with remarking that the Cayley transform $c: \mathcal{M} \rightarrow \mathbb{C}$

$$
c(z)=i \frac{z+i}{z-i}
$$

is an isometry of the unit disc onto the upper half plane which commutes with the Möbius actions.

The positive definite symmetric matrices. The example of the upper half plane can be generalized in higher dimensions. We have already seen that there exists a diffeomorphism between the upper half plane and $G / K$ where $G=\operatorname{SL}(2, \mathbb{R})$ and $K=\mathrm{SO}(2)$. We are going to investigate the case where $G=\mathrm{SL}(d, \mathbb{R}), d \geq 2$. We denote by $(\cdot, \cdot)$ the usual scalar product in $\mathbb{R}^{d}$ and we put

$$
\mathrm{P}(d, \mathbb{R}):=\left\{p \in \operatorname{Sym}(d):(v, p v)>0 \text { for every } v \in \mathbb{R}^{d}\right\},
$$

the set of $d \times d$ positive-definite symmetric matrices. Observe that $\mathrm{P}(d, \mathbb{R})$ is an open subset of $\operatorname{Sym}(d)$ and so it is naturally a smooth manifold. Its dimension is

$$
m:=\operatorname{dim}(\mathrm{P}(d, \mathbb{R}))=\frac{d(d+1)}{2} .
$$

We show that $\mathrm{P}(d, \mathbb{R}) \subseteq \mathbb{R}^{m}$ is the interior of a convex cone. Let $p, q \in \mathrm{P}(d, \mathbb{R})$ and $t>0$, then $t p \in \mathrm{P}(d, \mathbb{R}),(1-t) q \in \mathrm{P}(d, \mathbb{R})$ and also

$$
t p+(1-t) q \in \mathrm{P}(d, \mathbb{R})
$$

provided that $0 \leq t \leq 1$. The boundary of $\mathrm{P}(d, \mathbb{R})$ is the set of all singular positive semidefinite matrices. It is easy to see that $\mathrm{P}(d, \mathbb{R})$ is a foliated manifold in which each leaf is the preimage of a positive number through the determinant mapping. The preimage of 1 under the determinant mapping is denoted by

$$
\mathrm{SP}(d, \mathbb{R}):=\mathrm{P}(d, \mathbb{R}) \cap \mathrm{SL}(d, \mathbb{R}) .
$$

The group $\mathrm{GL}(d, \mathbb{R})$ acts on $\mathrm{P}(d, \mathbb{R})$ by the action

$$
(g, p) \mapsto g p^{t} g=: g[p]
$$




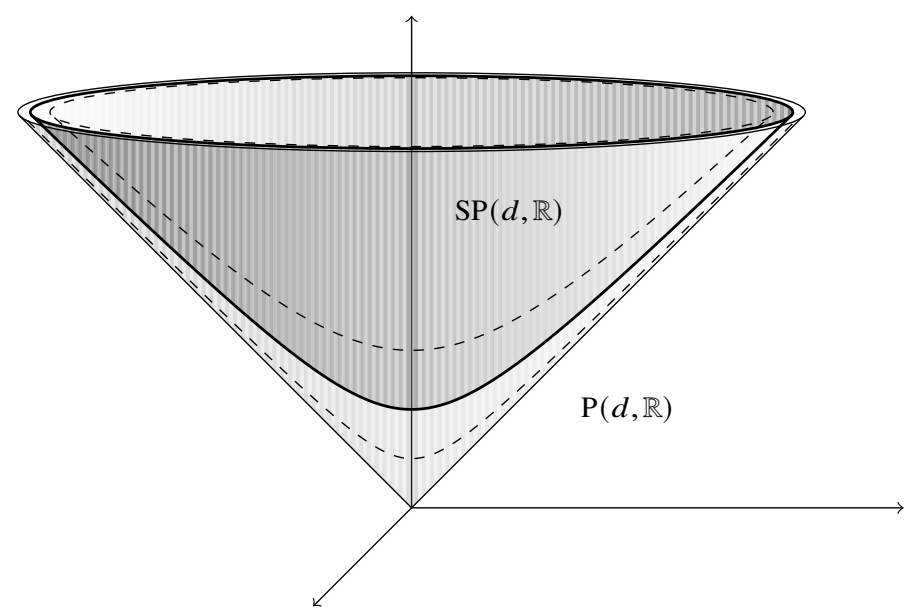

Fig. 1: The foliation of the cone $\mathrm{P}(2, \mathbb{R})$ consists of the connected components of the hyperboloids of two sheets each of which is the preimage under the determinant mapping of a positive number.

We next show that the action is transitive. By the spectral theorem, for every $p \in$ $\mathrm{P}(d, \mathbb{R})$ there exist $O \in \mathrm{SO}(d, \mathbb{R})$ and a diagonal matrix $D$ with positive entries on the diagonal such that $p=O^{-1} D O$. Since $D$ has positive entries on the diagonal, we can take its square root $D^{\frac{1}{2}}$. Let $g=O^{-1} D^{\frac{1}{2}} O$, then $g={ }^{t} g$ and

$$
p=g^{t} g=g\left[\mathrm{I}_{d}\right],
$$

which proves that the action is transitive. The stabilizer at $\mathrm{I}_{d} \in \mathrm{P}(d, \mathbb{R})$ is

$$
\mathrm{O}(d, \mathbb{R}):=\left\{g \in \mathrm{GL}(d, \mathbb{R}): g^{t} g=\mathrm{I}_{d}\right\} .
$$

Hence we have the diffeomorphism

$$
\mathrm{P}(d, \mathbb{R}) \simeq \mathrm{GL}(d, \mathbb{R}) / \mathrm{O}(d, \mathbb{R}) .
$$

The submanifold $\operatorname{SP}(d, \mathbb{R})$ is stable under the restriction of the previous action to $\mathrm{SL}(d, \mathbb{R})$, whose action on $\mathrm{SP}(d, \mathbb{R})$ is transitive. The stabilizer of $\mathrm{I}_{d}$ is $\mathrm{SO}(d, \mathbb{R})$, so

$$
\mathrm{SP}(d, \mathbb{R}) \simeq \operatorname{SL}(d, \mathbb{R}) / \mathrm{SO}(d, \mathbb{R}) .
$$

Now we analyze the Riemannian structure on $\mathrm{P}(d, \mathbb{R})$, using [25] as main reference. First of all, we observe that if $p \in \mathrm{P}(d, \mathbb{R})$, then $T_{p} \mathrm{P}(d, \mathbb{R}) \simeq \operatorname{Sym}(d)$. We define

$$
\langle X, Y\rangle_{p}:=\operatorname{tr}\left(p^{-1} X p^{-1} Y\right)
$$

where $X, Y \in T_{p}(\mathrm{P}(d, \mathbb{R}))$. It is easy to see that $\langle\cdot, \cdot\rangle_{p}$ is an inner product. We check that the $\operatorname{GL}(d, \mathbb{R})$-action preserves this form. Let $g \in \mathrm{GL}(d, \mathbb{R})$. Then by (8) and (9) 


$$
\begin{aligned}
\langle\mathrm{d} g(X), \mathrm{d} g(Y)\rangle_{g . p} & =\left\langle g X^{t} g, g X^{t} g\right\rangle_{g . p} \\
& =\operatorname{tr}\left({ }^{t} g^{-1} p^{-1} X p^{-1} Y^{t} g\right) \\
& =\operatorname{tr}\left(p^{-1} X p^{-1} Y\right)=\langle X, Y\rangle_{p},
\end{aligned}
$$

because the trace is invariant under conjugation. Hence the Riemannian structure on $\mathrm{P}(d, \mathbb{R})$ defined in $(9)$ is $\mathrm{GL}(d, \mathbb{R})$-invariant. Now, take $p \in \mathrm{P}(d, \mathbb{R})$ and define the mapping $\sigma_{p}: \mathrm{P}(d, \mathbb{R}) \rightarrow \mathrm{P}(d, \mathbb{R})$ by

$$
\sigma_{p}(q)=p q^{-1} p=p q^{-1 t} p .
$$

Clearly, $\sigma_{p}(p)=p$ and $\sigma_{p}^{2}(q)=q$ for every $q \in \mathrm{P}(d, \mathbb{R})$. It remains to show that $p$ is an isolated fixed point for $\sigma_{p}$. Let $q \in \mathrm{P}(d, \mathbb{R})$ be another nearby fixed point, that is $p q^{-1} p=q$. Thus, there exist $Y \in \mathfrak{g}$ and a small $t>0$ such that $q=p \exp (t Y)$. Hence

$$
p(p \exp (t Y))^{-1} p=p \exp (t Y),
$$

that is $\exp (-t Y)=\exp (t Y)$. If $t$ is smaller than the radius of the ball in which the exponential mapping is injective, this implies $Y=0$ and so $q=p$. We have proved that $\mathrm{P}(d, \mathbb{R})$ is a symmetric space. Observe that if $p \in \operatorname{SP}(d, \mathbb{R})$, then $\sigma_{p}(\operatorname{SP}(d, \mathbb{R}))=$ $\operatorname{SP}(d, \mathbb{R})$ and so $\operatorname{SP}(d, \mathbb{R})$ with the Riemannian metric restricted from $\mathrm{P}(d, \mathbb{R})$ is a symmetric space, too.

In the special case $d=2$, the symmetric space $\operatorname{SP}(2, \mathbb{R})$ is isomorphic to the unit disk, in fact it is one of the possible realizations of the hyperbolic space $\mathbb{H}^{1}$. It is important to observe that for a general $d>2$ there are no isometries between $\mathbb{H}^{d}=\mathrm{SO}(d, 1) / \mathrm{SO}(d)$ and $\mathrm{SP}(d, \mathbb{R})$, because the former has constant curvature while the latter has not.

The next results establish that there the Riemannian globally symmetric spaces are completely described by Lie algebraic data.

Proposition 1 (Lemma 3.2, Chap. IV, [15]). Let $\mathcal{M}$ be a Riemannian globally symmetric space. Then $I(\mathcal{M})$ has a smooth structure compatible with the compactopen topology which makes it a Lie group.

Theorem 3 (Theorem 3.3, Chap. IV, [15]). Let $\mathcal{M}$ be a Riemannian globally symmetric space, $p_{0} \in \mathcal{M}, G=I_{0}(\mathcal{M})$, the connected component of the identity of $I(\mathcal{M})$.

(i) The isotropy subgroup $K$ of $G$ at $p_{0}$ is compact, and $\mathcal{M} \simeq G / K$ under the map $g K \mapsto g\left[p_{0}\right]$.

(ii) The map $\sigma: g \mapsto s_{p_{0}} g s_{p_{0}}$ is an involutive automorphism of $G$ such that $K$ lies between the closed group $K_{\sigma}$ of the fixed points of $\sigma$ and its identity component. The subgroup $K$ contains no normal subgroups other than $\{e\}$.

(iii) Let $\mathfrak{g}$ be the Lie algebra of $G$ and $\mathfrak{f}$ be the Lie algebra of $K$. Then

$$
\mathfrak{f}=\left\{X \in \mathfrak{g}:\left(\mathrm{d} \sigma_{e}\right) X=X\right\}
$$


and if

$$
\mathfrak{p}=\left\{X \in \mathfrak{g}:\left(\mathrm{d} \sigma_{e}\right) X=-X\right\}
$$

then $\mathfrak{g}=\mathfrak{f}+\mathfrak{p}$ as vector space direct sum. Let $\pi$ denote the natural projection $G \rightarrow G / K$. Then $\mathrm{d} \pi_{e}$ maps $\mathfrak{f}$ into $\{0\}$ and $\mathfrak{p}$ isomorphically onto $T_{p_{0}} \mathcal{M}$. If $X \in \mathfrak{p}$, then the geodesic emanating from $p_{0}$ with tangent vector $\mathrm{d} \pi_{e}(X)$ is given by

$$
\gamma_{\mathrm{d} \pi_{e}(X)}(t)=\exp t X \cdot p_{0} .
$$

Moreover, if $Y \in T_{p_{0}} \mathcal{M}$, then $(\operatorname{dexp} t X)_{p_{0}} Y$ is the parallel translate of $Y$ along the geodesic.

Definition 3. Let $G$ be a connected Lie group and $H$ a closed subgroup. The pair $(G, H)$ is called a symmetric pair if there exists an involutive analytic automorphism $\sigma$ of $G$, briefly called an involution, such that

$$
(\operatorname{Fix}(\sigma))_{0} \subset H \subset \operatorname{Fix}(\sigma) .
$$

If in addition the group $\operatorname{Ad}_{G}(H)$ is compact, then $(G, H)$ is called a Riemannian symmetric pair.

Proposition 2 (Proposition 3.4 and Proposition 3.5, Chap. IV, [15]). Let $(G, K)$ be a Riemannian symmetric pair, $\pi: G \rightarrow G / K$ the projection, $o=\pi(e)$. Let $\sigma$ be any involution of $G$ such that $(\operatorname{Fix}(\sigma))_{0} \subset K \subset \operatorname{Fix}(\sigma)$. In each $G$-invariant Riemannian structure $Q$ on $G / K$, and such $Q$ do exist, the manifold $G / K$ is a Riemannian globally symmetric space. The geodesic symmetry $\sigma_{o}$ satisfies

$$
\sigma_{o} \circ \pi=\pi \circ \sigma, \quad \tau(\sigma(g))=\sigma_{o} \tau(g) \sigma_{o},
$$

where $\tau(g): G / K \rightarrow G / K$ is the natural action of $g$, namely $\tau(g) x K=g x K$. In particular $\sigma_{o}$ is independent of the choice of $Q$. Finally, if $z$ is the Lie algebra of the center of $G$ and $\mathfrak{k} \cap \mathfrak{z}=\{0\}$, then there exists exacly one involution $\sigma$ of $G$ such that $(\operatorname{Fix}(\sigma))_{0} \subset K \subset \operatorname{Fix}(\sigma)$.

The previous two results may be condensed in the statement that there is a bijective correspondence between Riemannian globally symmetric spaces and Riemannian symmetric pairs.

\subsection{Types of Symmetric Spaces}

The next step in the general theory of symmetric spaces is to look at the Lie algebra level. This is suggested by Theorem 3, which shows that a Riemannian globally symmetric space gives rise to a pair $(\mathfrak{g}, s)$, where $s=\mathrm{d} \sigma_{e}$, that satisfies

(i) $\mathfrak{g}$ is a real Lie algebra;

(ii) $s$ is an involutive automorphism of $\mathfrak{g}$; 
(iii) the fixed points $\mathfrak{f}$ of $s$ form a Lie algebra compacly contained in $\mathfrak{g}$,

where (iii) holds because $K$ is compact (see Chap. II in [15] for the definition of compactly embedded Lie subalgebra).

A pair ( $\mathfrak{g}, s$ ) satisying (i), (ii), and (iii) above is called an orthogonal symmetric Lie algebra. If in addition

(iv) $\mathfrak{f} \cap \mathfrak{z}=\{0\}$,

then $(\mathfrak{g}, s)$ is called effective. Fix such a pair and consider the decomposition $\mathfrak{g}=\mathfrak{u}+\mathfrak{e}$ into the +1 and -1 eigenspaces with respect to $s$. Motivated by the important decomposition result stated below in Theorem 4, one introduces the following terminology:

(a) if $\mathfrak{g}$ is compact and semisimple, then $(\mathfrak{g}, s)$ is said to be of the compact type;

(b) if $\mathfrak{g}$ is noncompact and semisimple and if $\mathfrak{g}=\mathfrak{u}+\mathfrak{e}$ is a Cartan decomposition, then $(\mathfrak{g}, s)$ is said to be of the noncompact type;

(c) if $\mathfrak{e}$ is an Abelian ideal in $\mathfrak{g}$, then $(\mathfrak{g}, s)$ is said to be of the Euclidean type.

Theorem 4 (Theorem 1.1, Chap. V, [15]). Suppose that $(\mathfrak{g}, s)$ is an effective orthogonal symmetric Lie algebra. Then there exist ideals $\mathfrak{g}_{0}, \mathfrak{g}_{-}$and $\mathfrak{g}_{+}$such that

(i) $\mathfrak{g}=\mathfrak{g}_{0}+\mathfrak{g}_{-}+\mathfrak{g}_{+}$, a Lie algebra direct sum;

(ii) $\mathfrak{g}_{0}, \mathfrak{g}_{-}$and $\mathfrak{g}_{+}$are invariant under $s$ and orthogonal with respect to the Killing form;

(iii) the pairs $\left(\mathfrak{g}_{0}, s_{0}\right),\left(\mathfrak{g}_{+}, s_{+}\right)$and $\left(\mathfrak{g}_{-} s_{-}\right)$are effective orthogonal symmetric Lie algebras of the Euclidean, compact and noncompact type, respectively.

The involutions $s_{0}, s_{-}$and $s_{+}$are those that arise by restricting $s$ to the corresponding ideals. The above result is of course of central importance because it allows to study separately the various cases. Clearly, the decomposition yields a corresponding decomposition of a symmetric space and thus induces the notions of symmetric space of Euclidean, compact and noncompact types. The Euclidean space, the sphere and the unit disk, introduced in Sect. 3.1, are the prototypical examples of such spaces. There is a remarkable duality between compact and noncompact types in which we are not interested. We content ourselves with mentioning that the compact types have positive sectional curvature and the noncompact ones have negative sectional curvature.

Since we are only interested in noncompact globlally symmetric spaces, we focus on the corresponding structural assumptions. To this end, we need yet another piece of terminology and we also slightly change the current notation to tune into the noncompact case. Any pair $(G, K)$ where $G$ is a connected Lie group with Lie algebra $\mathfrak{g}$ and where $K$ is a Lie subgroup of $G$ with Lie algebra $\mathfrak{f}$ is said to be associated to the (effective) orthogonal symmetric Lie algebra $(\mathfrak{g}, \theta)$, and will be called of the noncompact type if such is $(\mathfrak{g}, \theta)$. Thus, from now on we fix an effective orthogonal symmetric Lie algebra $(\mathfrak{g}, \theta)$ of the noncompact type, so that the eigenspace decomposition relative to $\theta$, namely $\mathfrak{g}=\mathfrak{f}+\mathfrak{p}$, is a Cartan decomposition. The next result is a cornerstone in the theory. 
Theorem 5 (Theorem 1.1, Chap. VI, [15]). With the notation above, suppose that $(G, K)$ is any pair associated with the effective orthogonal symmetric Lie algebra of the noncompact type $(\mathfrak{g}, \theta)$. Then:

(i) $K$ is connected, closed and contains the center $Z$ of G. Moreover, $K$ is compact if and only if $Z$ is finite. In this case, $K$ is a maximal compact subgroup of $G$;

(ii) there exists an involutive analytic automorphism $\Theta$ of $G$ whose fixed point set is $K$ and whose differential at the identity $e \in G$ is $\theta$; the pair $(G, K)$ is a Riemannian symmetric pair;

(iii) the mapping $\varphi:(X, k) \mapsto(\exp X) k$ is a diffeomorphism of $\mathfrak{p} \times K$ onto $G$ and the mapping Exp is a diffeomorphism of $\mathfrak{p}$ onto the globally symmetric space $G / K$.

The exponential mapping Exp in item (iii) above, quoted for completeness, is just the Riemannian exponential mapping (see for instance [15]) and will play no explicit role in what follows.

Assumption 1. From now on, let $G$ be a connected semisimple Lie group with finite center and $X=G / K$ the associated symmetric space of the noncompact type, where $K$ is a maximal compact subgroup of $G$. We also fix an Iwasawa decomposition $G=K A N$ and we denote by $M$ the centralizer of $A$ in $K$.

\subsection{Boundary of a Symmetric Space}

Our basic example of noncompact symmetric space will be the unit disk $\mathbb{D}$, which has a rather obvious (topological) boundary, namely the unit circle $S^{1}=\{z \in \mathbb{C}:|z|=1\}$. The notion of boundary of a symmetric space is highly non-trivial. For a deep study on the matter, the reader is referred to the classical paper of Furstenberg [11] in which a detailed motivation of Definition 4 below may be found. For our purposes, some heuristics and some basic observations will suffice.

Notice first that the Möbius action of $G=\mathrm{SU}(1,1)$ on $\mathbb{C}$ has precisely three orbits, namely $\mathbb{D}, S^{1}$ and the complement $\{w \in \mathbb{C}:|w|>1\}$. We already know that $\mathbb{D}$ is an orbit. Further, $A N$ fixes 1 (easy to check) and $K$ moves it along the unit circle, so that the $G$-orbit of 1 is $S^{1}$. Finally, for $\rho>1$ the formula

$$
k_{\theta / 2} \cdot \rho=\left[\begin{array}{cc}
\mathrm{e}^{i \theta / 2} & 0 \\
0 & \mathrm{e}^{-i \theta / 2}
\end{array}\right] \cdot \rho=\rho \cos \theta+i \rho \sin \theta,
$$

shows that $K$ maps the point $\rho$ along the circle of radius $\rho$ and any such real point may be reached, say, from 2 by means of $A$ because for $t>0$ the real numbers

$$
a_{t}[2]=\left[\begin{array}{c}
\cosh t \sinh t \\
\sinh t \cosh t
\end{array}\right][2]=\frac{2+\tanh t}{2 \tanh t+1}
$$

span the half-line $(1,+\infty)$. Thus the set $\{w \in \mathbb{C}:|w|>1\}$ is an orbit.

Let's go back to the unit circle. As already noticed, $A N$ fixes 1 and $K$ moves it along the circle, as can also be deduced from (10) when $\rho=1$. The very same 
formula shows also that the elements $k_{\theta / 2}$ when $\theta$ is any multiple of $2 \pi$ fix 1 . These are $\pm I$, namely the elements of $M$, the centralizer of $A$ in $K$. Therefore, the stabilizer of 1 is the group $P=M A N$ and $S^{1} \simeq G / P$. By means of the Iwasawa decomposition we may write

$$
S^{1} \simeq K A N / M A N
$$

and the natural question arises whether this is the same as $K / M$ or not. In the case at hand this is quite clearly so because $K$ acts transitively with isotropy $M$. This actually holds more generally in the sense that

$$
G / P=K A N / M A N \simeq K / M .
$$

Indeed, $K$ acts on the coset space $G / P$ in the natural fashion $k \cdot g P=(\mathrm{kg}) P$ and by the Iwasawa decomposition $k \in P=M A N$ if and only if $k \in M$. Hence the isotropy at the coset $\{P\}$ is $M$. Further, again by the Iwasawa decomposition, the action is transitive, and we conclude that $G / P \simeq K / M$. The reverse point of view (that of $G$ acting on $K / M$ with isotropy $P$ ) will be illustrated below in (15), where the explicit action of $G$ on $K / M$ is given.

Definition 4. The boundary of $X$ is the coset space $B:=K / M$.

We remark here en passant that $M$, which will play an important role below, normalizes $N$, that is

$$
m N m^{-1}=N, \quad m \in M .
$$

To see this, look at the Lie algebra level. If $\alpha$ is a positive root and $X \in \mathfrak{g}_{\alpha}$, then for every $H \in \mathfrak{a}$ it is

$$
[H, \operatorname{Ad} m X]=\operatorname{Ad} m\left[\operatorname{Ad} m^{-1} H, X\right]=\operatorname{Ad} m[H, X]=\alpha(H) \operatorname{Ad} m X,
$$

so that $\operatorname{Ad} m\left(\mathfrak{g}_{\alpha}\right) \subset \mathfrak{g}_{\alpha}$. An other normalization property that involves $N$ is that for any $\alpha \in A$ and any $v \in N$ it holds

$$
\alpha v a N=a N \alpha v .
$$

This, in turn, follows from choosing $v^{\prime} \in N$ such that $v^{\prime} \alpha=\alpha v$, which gives

$$
\alpha v a N=\alpha a a^{-1} v a N=\alpha a N \alpha^{-1} \alpha=a \alpha N \alpha^{-1} \alpha=a N \alpha=a N v^{\prime} \alpha=a N \alpha \nu .
$$

\subsection{Changing the Reference Point}

In what follows, it will be useful to change the reference point of both the symmetric space $X$ and its boundary. Although conceptually very well known and somehow trivial, the actual explicit determination of what happens when doing so is not to be found in the literature, to the best of our knowledge. In order to see how the various decompositions are affected by changing the origin of our spaces, it is convenient to 
introduce Borel sections and occasionaly adopt a slightly different notation for the (various) $G$-actions.

The action of $G$ on $X=G / K$ will be written $g[x]$, namely

$$
g[x]=g[h K]=g h K .
$$

For any fixed $x_{0} \in X=G / K$, a Borel section relative to $x_{0}$ is a measurable map $s_{x_{0}}: X \rightarrow G$ satisfying $s_{x_{0}}(x)\left[x_{0}\right]=x$ and $s_{x_{0}}\left(x_{0}\right)=e$, with $e$ the neutral element of $G$. Borel sections always exist since $G$ is second countable, see Theorem 5.11 in [26].

We next show how, in the present context, a Borel section associated to $o=e K \in$ $G / K$ can be determined quite explicitly. Since $K$ is the isotropy subgroup of $G$ at $o$, the map $\beta: g K \mapsto g[o]$ is a diffeomorphism of $G / K$ onto $X$. Furthermore, by the Iwasawa decomposition of $G$ (Theorem 1), each element of $g \in G$ can be written as the product $g=n a k$ for exactly one triple $(n, a, k) \in N \times A \times K$, and the correspondence $(n, a, k) \leftrightarrow n a k$ is a diffeomorphism with $G$. Hence each class in $G / K$ has a representative of the form $n a K$ with unique $a \in A$ and $n \in N$, so that the mapping $\psi: G / K \rightarrow N A$ given by $n a K \mapsto n a$ is a diffeomorphism. It follows that the measurable, actually smooth, map

$$
\psi \circ \beta^{-1}: X \longrightarrow N A
$$

is a Borel section. Indeed, $\psi \circ \beta^{-1}(o)=\psi(K)=e$ and, by construction, for every $x \in X$, it holds $\psi \circ \beta^{-1}(x)[o]=x$. From now on, we will denote by $s_{o}$ the Borel section $\psi \circ \beta^{-1}$ with image $N A \subseteq G$.

Fix now $x \in X$ and let $K_{x}$ be the isotropy of $G$ at $x \in X$. Evidently,

$$
K_{x}=s_{o}(x) K s_{o}(x)^{-1} .
$$

It is then possible to write an Iwasawa decomposition w.r.t. the subgroup $K_{x}$. In fact,

$$
G=s_{O}(x) G s_{O}(x)^{-1}=s_{O}(x) K A N s_{o}(x)^{-1}=s_{O}(x) K s_{o}(x)^{-1} A N=K_{x} A N,
$$

because, as observed earlier, $s_{o}(x) \in A N$. By using the same approach, one obtains the various versions of the Iwasawa decomposition where the factors appear in a different order. It is worth observing that the subgroups $A$ and $N$ are independent of the maximal compact subgroup $K_{x}$, but the individual factors appearing in the decomposition of a fixed element $g \in G$ are not. Given $g \in G$, we denote with $H_{x}(g)$, $A_{x}(g)$ the elements of $\mathfrak{a}$ uniquely determined by

$$
g \in K_{x} \exp H_{x}(g) N, \quad g \in N \exp A_{x}(g) K_{x}
$$

and by $\kappa_{x}(g)$ the unique element in $K_{x}$ such that $g \in \kappa_{x}(g) A N$. Clearly,

$$
A_{x}\left(g^{-1}\right)=-H_{x}(g)
$$


Once the point $x \in X$ has been fixed, a Borel section $s_{x}: X \rightarrow G$ can also be fixed, so that for every $y \in X, s_{x}(y)[x]=y$ and $s_{x}(x)=e$. As before, it may be arranged that $s_{x}(y) \in N A=A N$. Also, we denote by $M_{x}$ the centralizer of $A$ in $K_{x}$, so that $M_{x}=s_{o}(x) M s_{o}(x)^{-1}$. The following technical observation will be useful below.

Lemma 1. For any $x \in X$ it is

(i) $\left.\kappa_{o} \circ \kappa_{x}\right|_{K}=i d_{K}$; in particular, if $k_{x}=\kappa_{x}(k)$ for some $k \in K$, then $k=\kappa_{o}\left(k_{x}\right)$;

(ii) $\left.\kappa_{x} \circ \kappa_{o}\right|_{K_{x}}=i d_{K_{x}}$.

Proof. We start by proving (i). Let $k \in K$. Then according to the Iwasawa decomposition $K_{x} A N$ it is $k=\kappa_{x}(k) a n$, that is $\kappa_{x}(k)=k(a n)^{-1} \in K A N$. So that $\kappa_{o}\left(\kappa_{x}(k)\right)$ is precisely $k$, as desired. The proof of (ii) is analogous.

The action of $G$ on the boundary $B=K / M$ is induced by the decomposition $G / P=K A N / M A N$ in the sense that if $g \in G$ and $k M \in B$ then

$$
g\langle k M\rangle:=\kappa_{o}(g k) M .
$$

Consider now the action of $K_{x}$. By the definition (15) and by item (i) in Lemma 1, for any $k \in K$ it is

$$
\kappa_{x}(k)\langle M\rangle=\kappa_{o}\left(\kappa_{x}(k)\right) M=k M .
$$

Thus the action of $K_{x}$ on the boundary is transitive. Next, observe that an element $k_{x}=s_{o}(x) k s_{o}(x)^{-1}$ stabilizes $M \in K / M$ if and only if $\kappa_{o}\left(s_{o}(x) k s_{o}(x)^{-1}\right) \in M$, which means $s_{o}(x) k \in M A N$. This, together with the fact that $M$ normalizes $A N$, implies that $k \in M$, hence $k_{x} \in M_{x}$. Therefore the isotropy group of $K_{x}$ at $M$ is $M_{x}$. This shows that the map induced by $\kappa_{o}$ on $K_{x} / M_{x}$, which we denote $\kappa_{x, o}$, namely

$$
\kappa_{x, o}: K_{x} / M_{x} \rightarrow K / M, \quad k_{x} M_{x} \mapsto \kappa_{x, o}\left(k_{x} M_{x}\right):=\kappa_{o}\left(k_{x}\right) M,
$$

is a diffeomorphism. Furthermore, $k M$ and $\kappa_{x}(k) M_{x}$ determine the same boundary point, because by (16) $\kappa_{o}\left(\kappa_{x}(k)\right) M=k M$. By Lemma 1 the inverse of $\kappa_{x, o}$ is the map

$$
\kappa_{o, x}: K / M \rightarrow K_{x} / M_{x}, \quad k M \mapsto \kappa_{o, x}(k M):=\kappa_{x}(k) M_{x} .
$$

\subsection{Horocycles}

A hyperplane in $\mathbb{R}^{n}$ is orthogonal to a family of parallel lines. What is a reasonable analogue of this in, say, Riemannian geometry? Since geodesics are very natural generalizations of lines, a possible answer is given by a manifold that is orthogonal to families of parallel geodesics. In the context of symmetric spaces, such manifolds will be called horocycles, sometimes also horospheres.

Let us see what this idea leads to in the context of the unit disk, our basic example of noncompact symmetric space. The origin in $\mathbb{D}$ will be denoted $o$. If $\gamma:[a, b] \rightarrow \mathbb{D}$ 
is a smooth curve with $\gamma(a)=o$ and $\gamma(b)=x \in(-1,1)$ is a point on the real axis, then the simple inequality

$$
\frac{\dot{x}(t)^{2}}{\left(1-x(t)^{2}\right)^{2}} \leq \frac{\dot{x}(t)^{2}+\dot{y}(t)^{2}}{\left(1-x(t)^{2}-y(t)^{2}\right)^{2}}
$$

shows that straight real lines through the origin are geodesics. We observe en passant that since $\gamma_{0}(t)=(t x, 0)$ with $t \in[0,1]$ is such a straight line, then

$$
d(o, x)=L\left(\gamma_{0}\right)=\int_{0}^{1} \frac{|x|}{1-t^{2}|x|} \mathrm{d} t=\frac{1}{2} \log \frac{1+|x|}{1-|x|} .
$$

As we know, $G=\mathrm{SU}(1,1)$ acts by isometries via the Möbius action on $\mathbb{D}$. Such maps are conformal and map circles and lines into circles and lines. Hence the geodesics in $\mathbb{D}$ are circular arcs perpendicular to the boundary $|z|=1$. All circular arcs perpendicular to the same point at the boundary may be seen as parallel lines and thus a natural notion of horocycle in this context is that of circle tangent to the boundary (less the point on $S^{1}$ ) because such a circle is of course perpendicular to all the above parallel geodesics.

The circle through the origin and tangent to the boundary at $1 \in \mathbb{C}$ is therefore the prototype of horocycle. Observe that

$$
n_{s}[o]=\left[\begin{array}{cc}
1+i s & -i s \\
i s & 1-i s
\end{array}\right][o]=\frac{-i s}{1-i s}=\frac{s}{s+i}=\frac{s^{2}}{s^{2}+1}-i \frac{s}{s^{2}+1}
$$

and an easy calculation shows that these are precisely the points on the circle of radius $1 / 4$ centered at $1 / 2 \in \mathbb{C}$ that are contained in $\mathbb{D}$. Furthermore, as $s \rightarrow \pm \infty$ one gets the boundary point $b_{0}=1 \in \mathbb{C}$. We have obtained the basic horocycle, which will be denoted $\xi_{o}$, as the $N$-orbit $N[o]$.

Other horocycles tangent to $b_{0}$ are the orbits $N a_{t}[o]=a_{t} N[o]$ where of course

$$
a_{t}=\left[\begin{array}{l}
\cosh t \sinh t \\
\sinh t \cosh t
\end{array}\right]
$$

is any member of $A$ (recall that $A$ normalizes $N$ ). This is because

$$
a_{t}[o]=\tanh t \in(-1,1)
$$

parametrizes any other point on the geodesic line $(-1,1) \subset \mathbb{C}$ and an easy calculation shows that its $N$-orbit is just the circle through that point and tangent to $b_{0}$ (see Fig. 2 ). It is clear that by acting with the rotation group one gets all other horocycles, that is, all the circles in $\mathbb{D}$ tangent to the boundary. Thus, any other horocycle $\xi$ can be written in the form $k a \cdot \xi_{0}$ with $k \in K$ and $a \in A$. But this means

$$
\xi=(k a) N(k a)^{-1}(k a[o]),
$$




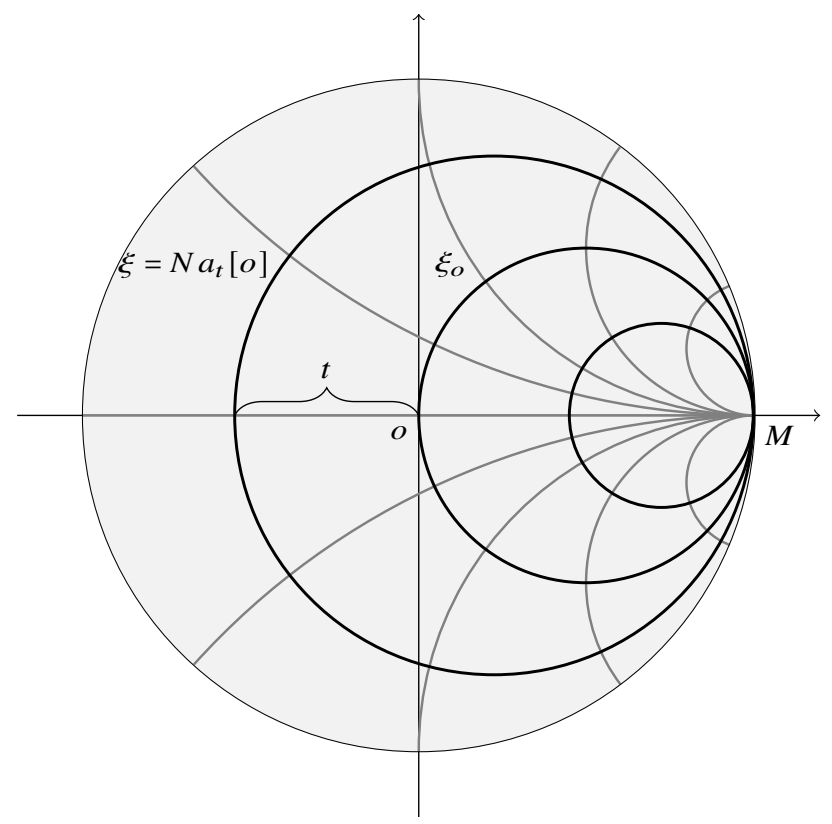

Fig. 2: The basic horocycle $\xi_{o}$ in the unit disc and the horocycle $\xi$ tangent to the boundary at 1 and with distance $-t$ from the origin $o$. In gray, the sheaf of parallel geodetics perpendicular to $\xi_{o}$ and $\xi$.

which exhibits $\xi$ as an orbit of a group conjugate to $N$, namely $(k a) N(k a)^{-1}$. This motivates the Definition 5 below.

Definition 5 ([17]). A horocycle in $X$ is any orbit of any subgroup of $G$ conjugate to $N$, that is an orbit $N^{g}[x]$ where $x \in X, g \in G$ and $N^{g}=g N g^{-1}$. We shall denote by $\Xi$ the set of all horocycles in $X$.

By Theorem 1.1 in Chap.II in [17], horocycles are closed submanifolds of $X$, the $G$-action on $X$ maps horocycles to horocycles and in fact the group $G$ acts transitively on $\Xi$ by

$$
\left(g, N^{h}[x]\right) \mapsto g .\left(N^{h}[x]\right):=g N^{h}[x] .
$$

We fix $x \in X$ and we consider the horocycle $\xi=N[x]$. By Theorem 1.1 in Chap.II in [17], the isotropy at $\xi$ is $M_{x} N$ and therefore

$$
\Xi \simeq G / M_{x} N
$$

under the diffeomorphism $g M_{x} N \mapsto g N[x]$. Furthermore, by Proposition 1.4 in Chap.II in [17], $\left(K_{x} / M_{x}\right) \times A$ is diffeomorphic to $G / M_{x} N$ under the mapping

$$
\left(k_{x} M_{x}, a\right) \mapsto k_{x} a M_{x} N
$$


Therefore, for each horocycle $\xi \in \Xi$ there exist unique $k_{x} M_{x} \in K_{x} / M_{x}$ and $a \in A$ such that

$$
\xi=k_{x} a N[x] .
$$

Finally, since $K / M$ is diffeomorphic to $K_{x} / M_{x}$ under the mapping $\kappa_{o, x}(k M)=$ $\kappa_{x}(k) M_{x}$, we define the diffeomorphism

$$
\Psi_{x}: K / M \times A \longrightarrow \Xi, \quad(k M, a) \mapsto \kappa_{x}(k) a N[x] .
$$

Observe that the boundary point $k M \in K / M$ which identifies the horocycle $\xi=$ $\kappa_{x}(k) a N[x]$ through (19) is independent of the choice of the reference point $x \in X$. Namely, for every $x, y \in X$

$$
\Psi_{x}(k M, a)=\Psi_{y}\left(k M, a^{\prime}\right)
$$

for some $a^{\prime} \in A$. Indeed, if $\xi=k_{x} a N[x]$ and if we pick $y \in X$, hence $k_{y} M_{y} \in K_{y} / M_{y}$ and $a^{\prime} \in A$ such that $\xi=k_{y} a^{\prime} N[y]$, then $k_{y} M_{y}=\kappa_{y}\left(k_{x}\right) M_{y}$ and this identifies the boundary point $\kappa_{o}\left(k_{x}\right) M$. Indeed, by the $K_{y} A N$ and $K A N$ Iwasawa decompositions of $k_{x}$, we have that

$$
\kappa_{y}\left(k_{x}\right) \in k_{x} A N=\kappa_{o}\left(k_{x}\right) A N,
$$

so that

$$
\kappa_{y, o}\left(\kappa_{y}\left(k_{x}\right) M_{y}\right)=\kappa_{o}\left(\kappa_{y}\left(k_{x}\right)\right) M=\kappa_{o}\left(k_{x}\right) M .
$$

We shall say that $\Psi_{x}(k M, a)$ represents the horocycle with normal $k M$ and composite distance $\log a$ from $x$ (see below, Definition 6). We stress that the normal of a horocycle is independent of the choice of $x \in X$. The composite distance, however, is different for different reference points.

This parametrization generalizes the geometric picture in $\mathbb{D}$, where a horocycle $\xi=k a_{t} N[o]$ is identified by the boundary point $k M \in K / M$ to which it is tangent and the "signed distance" $t$ from the reference point, see Fig. 2.

Proposition 3. Fix a reference point $x \in X$. The horocycle through $y \in X$ with normal $k M$ is $N^{\kappa_{x}(k)}[y]$.

Proof. An equivalent statement is that, writing $k=\kappa_{o}\left(k_{x}\right)$ with $k_{x} \in K_{x}$, the horocycle through $y$ with normal $\kappa_{o}\left(k_{x}\right) M$ is $k_{x} N k_{x}^{-1}[y]$ because $k_{x}=\kappa_{x}(k)$ by item (ii) in Lemma 1.

Since $k=\kappa_{o}\left(k_{x}\right)$, then $k M$ and $k_{x} M_{x}$ identify the same boundary point and a horocycle with normal $k M$ has the form $\xi=k_{x} a M_{x} N$ as in (18). If this represents a horocycle through $y$, then there exists $g \in G$ such that

$$
\xi=g N g^{-1}[y]=\kappa_{x}(g) N \kappa_{x}(g)^{-1}[y] .
$$

Now observe that there exist $\alpha \in A$ and $v \in N$ such that $\kappa_{x}(g)^{-1}[y]=v \alpha[x]$, then $\xi=\kappa_{x}(g) \alpha N[x]$. Thus, since $\xi=k_{x} a N[x]$, we have that

$$
\kappa_{x}(g) \alpha N[x]=k_{x} a N[x],
$$


which by (18) implies $\kappa_{x}(g) M_{x}=k_{x} M_{x}$. Hence $\kappa_{x}(g)=k_{x} m_{x}$ for some $m_{x} \in M_{x}$. However, (11) implies at once that $m_{x} N m_{x}^{-1}=N$, and hence $N^{K_{x}(g)}=N^{k_{x}}$.

Definition 6. Fix a reference point $x \in X$ and choose $y \in X$ and $b \in K / M$, so that by Proposition 3 the horocycle $\xi=\xi(y, b)$ passing through $y$ with normal $b=k M$ is uniquely determined, and hence there exists a unique $a \in A$ such that

$$
\xi(y, k M)=\kappa_{x}(k) a N[x] .
$$

We denote by $A_{x}(y, b) \in \mathfrak{a}$ the composite distance of the horocycle $\xi(y, b)$ from $x \in X$, namely

$$
A_{x}(y, b)=\log a,
$$

The reader is warned not to confuse the composite distance $A_{x}(y, b)$, which depends on $(y, b) \in X \times B$, with the Abelian component $A_{x}(g)$ of $g$ in the Iwaswawa decomposition $N A K_{x}$, which is a function on $G$ (see (13)). A relation between the two does exist, as pointed out in the next lemma, where we collect several properties of the composite distance which will play a crucial role in our work.

Lemma 2. Fix a reference point $x \in X$. Then:

(i) for any $k_{x} \in K_{x}$ and $g \in G$ we have

$$
A_{x}\left(g[x], \kappa_{o}\left(k_{x}\right) M\right)=A_{x}\left(k_{x}^{-1} g\right),
$$

where the right-hand side is defined by (13);

(ii) for any $y \in X, k M \in K / M$ and $g \in G$ we have

$$
A_{x}(y, k M)=A_{g[x]}(g[y], g\langle k M\rangle) ;
$$

(iii) for any $y, z \in X$ and $k M \in K / M$ we have

$$
A_{x}(y, k M)=A_{x}(z, k M)+A_{z}(y, k M) .
$$

Proof. (i) Let $k_{x} \in K_{x}$ and $g \in G$. By Proposition 3 and (ii) of Lemma 1, the horocycle passing through $g[x]$ with normal $\kappa_{o}\left(k_{x}\right) M$ is $k_{x} N k_{x}^{-1} g[x]$. By Definition 6, we have that

$$
k_{x} N k_{x}^{-1} g[x]=k_{x} \exp \left(A_{x}\left(g[x], \kappa_{o}\left(k_{x}\right) M\right)\right) N[x],
$$

and so $k_{x}^{-1} g \in N \exp \left(A_{x}\left(g[x], \kappa_{o}\left(k_{x}\right) M\right)\right) K_{x}$. This proves (i).

(ii) For simplicity, we first prove the statement in the case $x=o$. Let $y \in X, k M \in K / M$ and $g \in G$. By Proposition 3, and the fact that $A$ normalizes $N$, the horocycle passing through $g[y]$ with normal $g\langle k M\rangle=\kappa_{O}(g k) M$ (see (15)) is

$$
N^{\kappa_{o}(g k)} g[y]=\kappa_{O}(g k) N \kappa_{O}(g k)^{-1} g[y]=g k N(g k)^{-1} g[y] .
$$

By the diffeomorphism given in (18), there exist $h \in K_{g[o]}$ and $a \in A$ such that

$$
g k N k^{-1}[y]=\operatorname{haNg}[o],
$$


and thus, by definition

$$
a=\exp \left(A_{g[o]}(g[y], g\langle k M\rangle)\right) .
$$

We need to show that $a=\exp \left(A_{o}(y, k M)\right)$. Since $K_{g[o]}=g K g^{-1}$, we have $h=$ $g k_{1} g^{-1}$ for some $k_{1} \in K$ and we claim that

$$
k_{1} \kappa_{o}\left(g^{-1}\right) M=k M
$$

By (23) we have that

$$
k_{1} g^{-1} a N s_{o}(g[o])[o]=k_{1} g^{-1} a N g[o]=k N k^{-1}[y]=k N s_{o}\left(k^{-1}[y]\right)[o] .
$$

Since $s_{o}$ takes values in $A N$ and writing the $N A K$ decomposition of $g^{-1}$, there exist $a^{\prime}, a^{\prime \prime} \in A$ such that

$$
k_{1} \kappa_{o}\left(g^{-1}\right) a^{\prime} N[o]=k a^{\prime \prime} N[o] .
$$

Hence, by (18) we have that $k_{1} \kappa_{o}\left(g^{-1}\right) M=k M$, that is the claim (24). Therefore, for some $m \in M$ the right-hand side of (23) is

$$
\begin{aligned}
\operatorname{haNg}[o] & =\operatorname{gkm\kappa } \kappa_{o}\left(g^{-1}\right)^{-1} g^{-1} a N g[o] \\
& =\operatorname{gkmaN}\left(\kappa_{o}\left(g^{-1}\right)^{-1} g^{-1}\right) g[o] \\
& =\operatorname{gkmaN} \kappa_{o}\left(g^{-1}\right)^{-1}[o] \\
& =\operatorname{gkmaN}[o]=\operatorname{gkaN}[o]
\end{aligned}
$$

where in the second line we have used that $\kappa_{o}\left(g^{-1}\right)^{-1} g^{-1} \in A N$ and then (12). Summarizing, we have shown that

$$
g k N k^{-1} s_{o}(y)[o]=\operatorname{gkaN}[o] .
$$

By taking $e \in N$ on the left, there must be $n \in N$ such that $s_{o}(y)[o]=k a n[o]$, so that $(k a n)^{-1} s_{o}(y) \in K$, whence $k^{-1} s_{o}(y) \in K a n$. This shows that

$$
a=\exp \left(A_{o}\left(k^{-1} s_{o}(y)\right)\right)=\exp \left(A_{o}(y, k M)\right),
$$

where the second equality follows by item (i). This concludes (ii) in the case $x=o$. The general case follows by the latter. Indeed, by applying it with $s_{o}(x)$ and $g s_{o}(x)$, respectively in the first and the second equality, we obtain

$$
A_{x}(y, k M)=A_{o}\left(s_{o}(x)^{-1}[y], s_{o}(x)^{-1}\langle k M\rangle\right)=A_{g[x]}(g[y], g\langle k M\rangle) .
$$

(iii) For simplicity we start by proving it for $x=o$, the general case follows. Let $y, z \in X$ and $k M \in K / M$. By the definition of $s_{z}$, we have that $s_{z}(o)^{-1}=s_{o}(z)$ and $K=s_{z}(o) K_{z} s_{z}(o)^{-1}$. Observe that, by the $K_{z} A N$ Iwasawa decomposition of $k$ 


$$
s_{z}(o) k \in s_{z}(o) \kappa_{z}(k) A N=s_{z}(o) \kappa_{z}(k) s_{z}(o)^{-1} A N
$$

and then

$$
\kappa_{o}\left(s_{z}(o) k\right)=s_{z}(o) \kappa_{z}(k) s_{z}(o)^{-1} .
$$

Furthermore, $s_{y}(o) k \in K \exp \left(H_{o}\left(s_{y}(o) k\right)\right) N$, so that

$$
s_{z}(o) k k^{-1} s_{y}(o)^{-1} \in s_{z}(o) \kappa_{z}(k) s_{z}(o)^{-1} N \exp \left(H_{o}\left(s_{z}(o) k\right)-H_{o}\left(s_{y}(o) k\right)\right) K .
$$

Now, observe that by (14) and (i) it is possible to rewrite

$$
\begin{aligned}
H_{o}\left(s_{z}(o) k\right)-H_{o}\left(s_{y}(o) k\right) & =A_{o}\left(k^{-1} s_{y}(o)^{-1}\right)-A_{o}\left(k^{-1} s_{z}(o)^{-1}\right) \\
& =A_{o}\left(s_{y}(o)^{-1}[o], k M\right)-A_{o}\left(s_{z}(o)^{-1}[o], k M\right) \\
& =A_{o}(y, k M)-A_{o}(z, k M) .
\end{aligned}
$$

Hence, (25) becomes

$$
s_{z}(o) s_{y}(o)^{-1} \in s_{z}(o) \kappa_{z}(k) s_{z}(o)^{-1} N \exp \left(A_{o}(y, k M)-A_{o}(z, k M)\right) K,
$$

and by conjugating by $s_{z}(o)^{-1} \in A N$

$$
\begin{aligned}
s_{y}(o)^{-1} s_{z}(o) & \in \kappa_{z}(k) s_{z}(o)^{-1} N \exp \left(A_{o}(y, k M)-A_{o}(z, k M)\right) K s_{z}(o) \\
& =\kappa_{z}(k) N \exp \left(A_{o}(y, k M)-A_{o}(z, k M)\right) s_{z}(o)^{-1} K s_{z}(o) \\
& =\kappa_{z}(k) N \exp \left(A_{o}(y, k M)-A_{o}(z, k M)\right) K_{z},
\end{aligned}
$$

where in the first equality we use (12). Finally, we observe that $s_{y}(o)^{-1} s_{z}(o)=$ $s_{o}(y) s_{z}(o)=s_{z}(y)$ and then

$$
\kappa_{z}(k)^{-1} s_{z}(y) \in N \exp \left(A_{o}(y, k M)-A_{o}(z, k M)\right) K_{z} .
$$

Therefore, by item (i) of Lemma 1 and item (i) above

$$
A_{o}(y, k M)-A_{o}(z, k M)=A_{z}\left(\kappa_{z}(k)^{-1} s_{z}(y)\right)=A_{z}(y, k M) .
$$

This proves the case $x=o$. The general case trivially follows:

$$
\begin{aligned}
A_{x}(z, k M)+A_{z}(y, k M) & =A_{o}(z, k M)-A_{o}(x, k M)+A_{o}(y, k M)-A_{o}(z, k M) \\
& =A_{x}(y, k M)
\end{aligned}
$$

This finishes the proof of the lemma.

Let $x \in X$. By Definition 6, for every $(k M, a) \in K / M \times A$ and $z \in X$

$$
z \in \Psi_{x}(k M, a) \quad \Longleftrightarrow \quad A_{x}(z, k M)=\log a
$$

Then, by (26) together with (21) it follows that 


$$
\begin{aligned}
z \in g . \Psi_{x}(k M, a) & \Longleftrightarrow g^{-1}[z] \in \Psi_{x}(k M, a) \\
& \Longleftrightarrow \log a=A_{x}\left(g^{-1}[z], k M\right) \\
& \Longleftrightarrow \log a=A_{g[x]}(z, g\langle k M\rangle) \\
& \Longleftrightarrow z \in \Psi_{g[x]}(g\langle k M\rangle, a) .
\end{aligned}
$$

So that,

$$
g . \Psi_{x}(k M, a)=\Psi_{g[x]}(g\langle k M\rangle, a) .
$$

Furthermore, if $y \in X$, then by (26) and (22) we have that

$$
\begin{aligned}
z \in \Psi_{x}(k M, a) & \Longleftrightarrow \log a=A_{x}(z, k M) \\
& \Longleftrightarrow \log a=A_{x}(y, k M)+A_{y}(z, k M) \\
& \Longleftrightarrow \log \left(a \exp \left(-A_{x}(y, k M)\right)\right)=A_{y}(z, k M) \\
& \Longleftrightarrow z \in \Psi_{y}\left(k M, a \exp \left(A_{y}(x, k M)\right)\right),
\end{aligned}
$$

where in the last equivalence we use the equality $A_{y}(x, k M)=-A_{x}(y, k M)$, which follows immediately by (22). Hence, we have

$$
\left(\Psi_{y}^{-1} \circ \Psi_{x}\right)(k M, a)=\left(k M, a \exp \left(A_{y}(x, k M)\right)\right) .
$$

Positive definite symmetric matrices. We recall the example of the positive definite symmetric matrices in order to explicitly describe the horocycles. Recall that the semisimple group associated to the symmetric space of the positive definite symmetric matrices is $G=\operatorname{SL}(d, \mathbb{R})$. As we have already seen, the Iwasawa decomposition of $G$ is formed by $K=\mathrm{SO}(d)$, the subgroup $A$ of diagonal matrices with positive entries on the diagonal and the subgroup $N$ of the unit upper triangular matrix. Hence the principal horocycle is

$$
\xi_{0}=N\left[\mathrm{I}_{d}\right]=\left\{n^{t} n: n \in N\right\} .
$$

Let $a=\operatorname{diag}\left(e^{a_{1}}, \ldots, e^{a_{d}}\right) \in A$, then the horocycle obtained as the $N$-orbit of $a K \in$ $\operatorname{SP}(d, \mathbb{R})$ is the subset of $\operatorname{SP}(d, \mathbb{R})$ of matrices of the form

$$
\left\{e^{a_{i}+a_{j}} \sum_{k=\max (i, j)}^{d} n_{i, k} n_{j, k}\right\}_{i, j},
$$

for every choice of $d(d-1) / 2$ values $n_{i, j} \in \mathbb{R}$ with $j>i$, where $n_{i, i}=1$. The subgroup $\bar{N}=\Theta(N)$ coincides with the lower unit triangular matrices. The $\bar{N}$-orbit of a positive definite diagonal matrix $a K$ is the set of all the symmetric positive definite matrices having $a^{2}$ as diagonal matrix in the usual $L D^{t} L$ decomposition. Furthermore, for every $a \in A$, we have $\bar{N}[a]=\left(N\left[a^{-1}\right]\right)^{-1}$. It follows that the horocycle $N[a]$ is the subset of $\operatorname{SP}(d, \mathbb{R})$ of matrices having $a^{2}$ as diagonal matrix in the $U D^{t} U$ decomposition. 


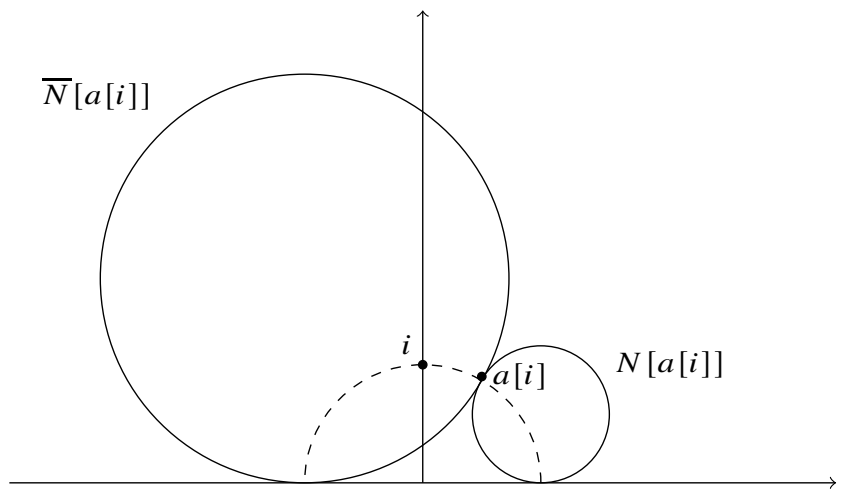

Fig. 3: In the special case $d=2$, the $N$-orbit and the $\bar{N}$-orbit are tangent circles. More in general, for any $w \in W$ the intersection between the $N$-orbit and the $\left(w N w^{-1}\right)$ orbit of a point coincides with the point itself, see Proposition 1.7 in Chap. II in [17].

Let $p \in \mathrm{SP}(d, \mathbb{R})$ and let $p=O D^{t} O$ be the spectral decomposition of $p$, with $O \in \mathrm{SO}(d)$ and $D$ diagonal matrix and let $k \in K$. Then we have

$$
k[p]=k p^{t} k=k O D^{t} O^{t} k
$$

and since $k O \in \mathrm{SO}(d)$ then $k[p]$ has the same eigenvalues of $p$. In fact, the $K$-orbit of $p \in \operatorname{SP}(d, \mathbb{R})$ is the subset of all the matrices in $\operatorname{SP}(d, \mathbb{R})$ with the same eigenvalues of $p$ and if $a \in A$ the matrix $k[a]$ has the columns of $k$ as eigenvectors. Furthermore in each $K$-orbit there exists a diagonal matrix with entries ordered decreasingly on the diagonal, that is a matrix that lies on $A_{+}\left[\mathrm{I}_{d}\right]$.

Finally, by (18), any horocycle $\xi \in \Xi$ can be written as $\xi=k a N\left[\mathrm{I}_{d}\right]$ for some $k \in K$ and $a \in A$. This is thus the subset of $\operatorname{SP}(d, \mathbb{R})$ of matrices having $a^{2}$ as diagonal matrix in the $U D^{t} U$ decomposition w.r.t. the $\mathbb{R}^{d}$-basis $\left\{k e_{i}\right\}_{i=1, \ldots, d}$, where $\left\{e_{i}\right\}_{i=1, \ldots, d}$ is the canonical basis of $\mathbb{R}^{d}$.

\section{Analysis on Symmetric Spaces}

We collect in this section the analytic ingredients that come into play. Apart from the basic measures and function spaces, we introduce the Helgason-Fourier transform and the Radon transform and recall the results that we use throughout. The main references are [16], [17]. 


\subsection{Measures}

This section is devoted to the measures that will be involved in what follows. We first present the Haar measure and then introduce the measures on the spaces $X, B$ and $\Xi$. These are necessary in order to define the function spaces that we are interested in, among which the $L^{2}$-spaces that carry the regular representations. General references are [8] for the first part, and [16] and [17] for the second.

\subsubsection{Haar measures and modular functions}

We recall some basic definitions and results of Analysis on locally compact groups. We shall use them in the more specific context of Lie groups. A standard reference is Chap. 2 in [8].

A topological group is a group $G$ endowed with a topology relative to which the group operations

$$
(g, h) \mapsto g h, \quad g \mapsto g^{-1}
$$

are continuous as maps $G \times G \rightarrow G$ and $G \rightarrow G$, respectively. $G$ is locally compact if every point has a compact neighborhood. We shall also assume our groups to be Hausdorff. In particular, all Lie groups are locally compact topological groups.

A Borel measure $\mu$ on the topological space $X$, that is, a meausure on the $\sigma$-algebra $\mathcal{B}(X)$ of the Borel sets of $X$, is called a Radon measure if:

(i) it is finite on compact sets;

(ii) it is outer regular on the Borel sets, that is for every Borel set $E$

$$
\mu(E)=\inf \{\mu(U): U \supset E, U \text { open } ;\}
$$

(iii) it is inner regular on the open sets, that is for every open set $U$

$$
\mu(U)=\sup \{\mu(K): K \subset E, K \text { compact }\} \text {. }
$$

Definition 7. A left Haar measure on the topological group $G$ is a non zero Radon measure $\mu$ such that $\mu(x E)=\mu(E)$ for every Borel set $E \subset G$ and every $x \in G$. Similarly for right Haar measures.

Of course, the prototype of Haar measure is the Lebesgue measure on the additive group $\mathbb{R}^{d}$, which is invariant under left (and right) translations. Compactly supported continuous functions on a topological space $Y$ are denoted $C_{c}(Y)$. An equivalent definition for the left Haar measure $\mu$ is to require that for every $f \in C_{c}(G)$ and $h \in G$,

$$
\int_{G} f(h g) \mathrm{d} \mu(g)=\int_{G} f(g) \mathrm{d} \mu(g) .
$$

A fundamental result on Haar measures is the following theorem due to A. Weil. 
Theorem 6 (Theorem 2.10, [8]). Every locally compact group $G$ has a left Haar measure $\lambda$, which is essentially unique in the sense that if $\mu$ is any other left Haar measure, then there exists a positive constant $C$ such that $\mu=C \lambda$.

If we fix a left Haar measure $\mu$ on $G$, then for any $g \in G$ the measure $\mu_{g}$ defined by

$$
\mu_{g}(E)=\mu(E g)
$$

is again a left Haar measure. Therefore there must exist a positive real number, denoted $\Delta(g)$ such that

$$
\mu_{g}=\Delta(g) \mu \text {. }
$$

The function $\Delta: G \rightarrow \mathbb{R}_{+}$is called the modular function. From now on, the choice of a left Haar measure $\mu$ is considered as implicitly made, and hence we write

$$
\mathrm{d} g:=\mathrm{d} \mu(g) .
$$

Proposition 4 (Proposition 2.24, [8]). Let $G$ be a locally compact group. The modular function $\Delta: G \rightarrow \mathbb{R}_{+}$is a continuous homomorphism into the multiplicative group $\mathbb{R}_{+}$. Furthermore, for every $f \in L^{1}(G, \mu)$ we have

$$
\int_{G} f(g h) \mathrm{d} g=\Delta(h)^{-1} \int_{G} f(g) \mathrm{d} g .
$$

A group for which every left Haar measure is also a right Haar measure, hence for which $\Delta \equiv 1$, is called unimodular. Large classes of groups are unimodular, such as the Abelian, compact, nilpotent, semisimple and reductive groups. Many solvable groups, however, are not. Prototypical examples of non unimodular groups are the Iwasawa $N A$ groups, such as the affine " $a x+b$ " group. A practical recipe for the computation of modular functions is given by the following proposition.

Proposition 5 (Proposition 2.30, [8]). If $G$ is a connected Lie group and $\mathrm{Ad}$ denotes the adjoint action of $G$ on its Lie algebra, then $\Delta(g)=\operatorname{det}\left(\operatorname{Ad}\left(g^{-1}\right)\right)$.

The basic spaces $X$ and $\Xi$ in which we are interested are homogeneous spaces of the same group $G$. From the point of view of Analysis, the natural question arises whether the homogeneous space $G / H$ admits a $G$-invariant Radon measure or not. The answer to this question is contained in Theorem 7 below, which relates integration on $G$ to an iterated integral, first on $H$ and then on $G / H$. These formulae are achieved by means of the natural projection operator $P: C_{c}(G) \rightarrow C_{c}(G / H)$, also known as Weil's mean opearator, defined by

$$
P f(g H)=\int_{H} f(g h) \mathrm{d} h,
$$

which is well defined by the left invariance of $\mathrm{d} h$, the Haar measure on $H$. Furthermore, it is possible to see that $P$ is continuous and surjective. We are now in a position to state this classical result, also known as the Weil's decomposition theorem. Here $\Delta_{G}$ and $\Delta_{H}$ are the modular functions of $G$ and $H$, respectively. 
Theorem 7 (Theorem 2.51, [8]). Let $G$ be a locally compact group and $H$ a closed subgroup. There is a G-invariant Radon measure $\mu$ on $G / H$ if and only if $\left.\Delta_{G}\right|_{H}=\Delta_{H}$. In this case, $\mu$ is unique up to a constant factor, and if the factor is suitably chosen then

$$
\int_{G} f(g) \mathrm{d} g=\int_{G / H} P f(g H) \mathrm{d} \mu(g H)=\int_{G / H} \int_{H} f(g h) \mathrm{d} h \mathrm{~d} \mu(g H),
$$

for every $f \in C_{c}(G)$.

Hence, there always exists a $G$-invariant Radon measure on $G / H$ whenever $H$ is compact, since $\left.\Delta_{G}\right|_{H}=\Delta_{H} \equiv 1$. Indeed, the image of $H$ under both modular functions is a compact subgroup of the multiplicative group of positive reals, namely $\{1\}$.

Although many homogeneous spaces do not admit invariant measures (for example $\mathbb{R}$ as a homogeneous space of the " $a x+b$ " group), all of them admit strongly quasi-invariant measures. If $\mu$ is a measure on $X=G / H$ and we write $\mu^{g}(E)=\mu(g E)$ for $E \in \mathcal{B}(X)$, we say that $\mu$ is a quasi-invariant measure if all the $\mu^{g}$ are equivalent, that is, mutually absolutely continuous. We say that $\mu$ is strongly quasi-invariant if there exists a continuous function $\lambda: G \times G / H \rightarrow(0,+\infty)$ such that

$$
\mathrm{d} \mu^{g}(x)=\lambda(g, x) \mathrm{d} \mu(x), \quad x \in X, g \in G .
$$

In other words, the requirement is that the Radon-Nikodym derivative $\left(\mathrm{d} \mu^{g} / \mathrm{d} \mu\right)(x)$ is jointly continuous in $g$ and $x$. As mentioned, all homogeneous spaces admit strongly quasi-invariant measures (see Proposition 2.56 and Theorem 2.58 in [8]).

\subsubsection{Measures on semisimple Lie groups of the noncompact type}

Let $G$ a semisimple Lie group. By Theorem 6, there exists a (left) Haar measure on $G$, unique up to multiplication by a positive constant. We recall that by Theorem 1 there exist subgroups $K, A$, and $N$ of $G$ such that $G=K A N=N A K$. Since each subgroup carries a Haar measure, the natural question arises whether it is possible to write the Haar measure of $G$ using the Haar measures of the three subgroups involved, which are all, individually, unimodular.

Since $K$ is compact, we normalize its Haar measure in such a way that the total measure is 1 . The Haar measure on $A$ is obtained by starting from the (positive) measure that any Riemannian manifold inherits from its metric, see e.g. Chap. I in [16]. The invariant metric is obtained by taking the restriction to $\mathfrak{a} \times \mathfrak{a}$ of the Killing form, which is positive definite on $\mathfrak{p} \times \mathfrak{p} \supset \mathfrak{a} \times \mathfrak{a}$, whereby $\mathfrak{a}$ is identified with the tangent space to $A$ at the identity. The standard normalization is to multiply the Riemannian measure by $(2 \pi)^{-\ell / 2}$, where $\ell=\operatorname{dim} A$. As for $N$, we normalize its Haar measure $\mathrm{d} n$ so that

$$
\int_{\bar{N}} e^{-2 \rho(H(\bar{n}))} \mathrm{d} \bar{n}=1,
$$


where $\bar{N}=\Theta(N)$ and $\mathrm{d} \bar{n}$ is the pushforward of $\mathrm{d} n$ under $\Theta$. The convergence of the above integral is no trivial matter, and is discussed in detail in [16].

Proposition 6 (Proposition 5.1, Chap. I, [16]). Let $\mathrm{d} k$, $\mathrm{d} a$ and $\mathrm{d} n$ be left-invariant Haar measures on $K, A$ and $N$, respectively. Then the left Haar measure $\mathrm{d} g$ on $G$ can be normalized so that

$$
\begin{aligned}
\int_{G} f(g) \mathrm{d} g & =\int_{K \times A \times N} f(\text { kan }) e^{2 \rho \log a} \mathrm{~d} k \mathrm{~d} a \mathrm{~d} n \\
& =\int_{N \times A \times K} f(\text { nak }) e^{-2 \rho(\log a)} \mathrm{d} n \mathrm{~d} a \mathrm{~d} k \\
& =\int_{A \times N \times K} f(\text { ank }) \mathrm{d} a \mathrm{~d} n \mathrm{~d} k
\end{aligned}
$$

for every $f \in C_{c}(G)$.

The case of the group $A N$ deserves a separate comment. We recall by Sect. 2 that $A N$ is in fact a semidirect product since $A$ acts on $N$ by conjugation. Furthermore, for any $H \in \mathfrak{a}$ and any root vector $X_{\alpha} \in \mathfrak{g}_{\alpha}$ it holds

$$
\operatorname{Ad}(\exp H)\left(X_{\alpha}\right)=\mathrm{e}^{\operatorname{ad} H}\left(X_{\alpha}\right)=\sum_{0}^{\infty} \frac{(\operatorname{ad} H)^{k}}{k !} X_{\alpha}=\mathrm{e}^{\alpha(H)} X_{\alpha} .
$$

It follows that upon choosing a basis of $m_{\alpha}$ root vectors for each positive root $\alpha$ it is

$$
\left.\operatorname{det} \operatorname{Ad}(\exp H)\right|_{\mathfrak{n}}=\prod_{\alpha>0} \mathrm{e}^{m_{\alpha} \alpha(H)}
$$

or, using (6),

$$
\left.\operatorname{det} \operatorname{Ad} a\right|_{\mathfrak{n}}=\mathrm{e}^{2 \rho(\log a)} .
$$

Proposition 5 now entails that the modular function of the $A N$ Iwasawa group is

$$
\Delta(n a)=\mathrm{e}^{-2 \rho(\log a)} .
$$

Indeed, in the computation of $\operatorname{det} \operatorname{Ad}(n a)$ on $\mathfrak{n}+\mathfrak{a}$, all is relevant is the action of $\operatorname{Ad} a$ on $\mathfrak{n}$ because the action of $\operatorname{Ad} a$ is unimodular on $\mathfrak{a}$ since $A$ is Abelian, the action of $\operatorname{Ad} n$ is unimodular on $\mathfrak{n}$ because $N$ is nilpotent and that of $\operatorname{Ad} n$ on $\mathfrak{a}$ is again unimodular because its projection on $\mathfrak{a}$ is the identity (see also Cor. 5.2 in Chap. I in [16]).

\subsubsection{Measures on $X$}

In order to do Analysis on the symmetric space $X$ it is important to introduce some basic functions spaces and differential operators. The reader is referred to Chap. II in [16]. 
A quick way to introduce differential operators on $X$ is to say that $D$ is such an operator if it is a linear mapping of $C_{c}^{\infty}(X)$ that decreases supports. Such operators have local nature, in the sense that it is possible to find for any coordinate patch $(\mathcal{U}, \phi)$ in $X$ and any open set $\mathcal{W}$ with compact closure in $\mathcal{U}$ a finite number of smooth functions $a_{\alpha}$ on $\mathcal{W}$ such that

$$
D f=\sum_{\alpha} a_{\alpha}\left(D^{\alpha}\left(f \circ \phi^{-1}\right)\right) \circ \phi
$$

for any $f \in C^{\infty}(\mathcal{W})$, where

$$
D^{\alpha}=\frac{\partial^{|\alpha|}}{\partial x_{1}^{\alpha_{1}} \partial x_{2}^{\alpha_{2}} \ldots \partial x_{d}^{\alpha_{d}}}
$$

is the standard partial derivative operator in $\mathbb{R}^{d}$ associated with the multi-index $\alpha \in \mathbb{Z}_{+}^{d}$. Because of this local nature, it is then possible to extend any differential operator $D$ to $C^{\infty}(X)$.

On any differentiable manifold, hence on a symmetric space $X$, two are the most relevant spaces to consider if distribution theory is among the desirable targets. These are the space of smooth complex valued functions $\mathcal{E}(X)$ on $X$ and the space $\mathcal{D}(X)$ of smooth complex valued functions with compact support on $X$. When this notation, due to Schwartz, is adopted, it is meant that these vector spaces are endowed with suitable topologies, see Chap. II in [16] for the details. We stress that in our analysis the topologies on $\mathcal{E}(X)$ and $\mathcal{D}(X)$ do not enter into play.

Now, our purpose is to determine an explicit $G$-invariant measure on the symmetric space $X=G / K$, whose existence is guaranteed by the fact that $K$ is compact (see the comment after Theorem 7). Recall that, by Proposition 6, if $g=n a k$, then the Haar measure of $G$ can be normalized so that

$$
\mathrm{d} g=e^{-2 \rho(\log a)} \mathrm{d} n \mathrm{~d} a \mathrm{~d} k,
$$

where $\mathrm{d} k, \mathrm{~d} a$, and $\mathrm{d} n$ are the Haar measures on $K, A$ and $N$ that have been fixed in the previous paragraph.

We endow $X$ with the $G$-invariant measure $\mathrm{d} x$ obtained as the pushforward of $\mathrm{d} g$ under the canonical projection $G \rightarrow G / K$. Thus, for any smooth compactly supported function $f \in \mathcal{D}(X)$

$$
\int_{X} f(x) \mathrm{d} x=\int_{G} f(g[o]) \mathrm{d} g=\int_{N A} f(n a[o]) e^{-2 \rho(\log a)} \mathrm{d} n \mathrm{~d} a .
$$

We henceforth denote by $L^{2}(X)$ the Lebsegue space of square integrable (equivalence classes of) functions with respect to this measure. The quasi-regular representation $\pi$ of $G$ on $L^{2}(X)$ is then defined in the usual way, namely

$$
\pi(g) f(x):=f\left(g^{-1}[x]\right), \quad f \in L^{2}(X), g \in G .
$$


It is a unitary non-irreducible representation. Actually, it is possible to construct a family of Hilbert spaces in which $L^{2}(X)$ can be decomposed as a direct integral, whereby the restriction of $\pi$ to each of them is irreducible. These are the spherical principal series representations, discussed in Chap. VI in [17]. It is also well known that $\pi$ is not square integrable.

\subsubsection{Measures on the Boundary}

We shall now define positive measures on the boundary $B$ using its various possible parametrizations. Since $K$ and $M$ are compact subgroups of $G$, there exists a probability $K$-invariant measure $\mu^{o}$ on $B=K / M$, see the comment below Theorem 7 . The choice of this measure is such that Weil's decomposition holds, assuming that we normalize the Haar measure of $M$ in such a way that the total measure is 1 . For every other choice of the reference point $x \in X$ the analogous objects $K_{x}, M_{x}$ and $\mu^{x}$ can be introduced. The relation between $\mu^{o}$ and $\mu^{x}$ can be determined explicitly. We consider the diffeomorphism $T_{x}: K \rightarrow K_{x}$ defined by $k \mapsto s_{o}(x) k s_{o}(x)^{-1}$. Its restriction to $M$ is a diffeomorphism between $M$ and $M_{x}$. Hence, $T_{x}$ induces the diffeomorphism $\tilde{T}_{x}: K / M \rightarrow K_{x} / M_{x}$ defined by

$$
\tilde{T}_{x}(k M)=T_{x}(k) M_{x}=s_{o}(x) k s_{o}(x)^{-1} M_{x}=s_{o}(x) k M s_{o}(x)^{-1} .
$$

Let $\left(\tilde{T}_{x}\right)_{*}\left(\mu^{o}\right)$ be the pushfoward of the measure $\mu^{o}$ under $\tilde{T}_{x}$. Clearly, $\left(\tilde{T}_{x}\right)_{*}\left(\mu^{o}\right)$ is a $K_{x}$-invariant probability measure on $K_{x} / M_{x}$ and therefore $\mu^{x}=\left(\tilde{T}_{x}\right)_{*}\left(\mu^{o}\right)$. As we saw in (16), $K_{x} / M_{x}$ is diffeomorphic to the boundary $K / M$ through the map induced by $\kappa_{o}$. Therefore, we can consider the following $K_{x}$-invariant probability measure on the boundary $B=K / M$

$$
v^{x}:=\left(\kappa_{o}\right)_{*}\left(\mu^{x}\right) .
$$

It is worth observing that $v^{o}=\mu^{o}$ and the following relation follows

$$
v^{x}=\left(\kappa_{o} \circ \tilde{T}_{x}\right)_{*}\left(v^{o}\right) .
$$

Lemma 3. The measure $v^{o}$ is $G$-quasi-invariant. Let $F \in C(K / M)$ and $g \in G$,

$$
\int_{K / M} F\left(g^{-1}\langle k M\rangle\right) \mathrm{d} v^{o}(k M)=\int_{K / M} F(k M) e^{-2 \rho\left(H_{o}(g k)\right)} \mathrm{d} v^{o}(k M) .
$$

Proof. By Lemma 5.19 in Chap.I in [17], for every $H \in C(K)$ and $g \in G$,

$$
\int_{K} H\left(\kappa_{o}\left(g^{-1} k\right)\right) \mathrm{d} k=\int_{K} H(k) e^{-2 \rho\left(H_{o}(g k)\right)} \mathrm{d} k .
$$

A function $F \in C(K / M)$ will now be regarded as an $M$-right invariant continuous function on $K$. By our choice of $v^{o}$, Theorem 7 holds and hence 


$$
\begin{aligned}
\int_{K} F(k) \mathrm{d} k & =\int_{K / M} \int_{M} F(k M m) \mathrm{d} m \mathrm{~d} v^{o}(k M) \\
& =\int_{K / M} F(k M) \int_{M} \mathrm{~d} m \mathrm{~d} v^{o}(k M) \\
& =\int_{K / M} F(k M) \mathrm{d} v^{o}(k M),
\end{aligned}
$$

where we have used the normalization of the Haar measure of $M$. The function $k \mapsto F\left(g^{-1}\langle k\rangle\right)=F\left(\kappa_{o}\left(g^{-1} k\right)\right)$ is $M$-invariant by $\kappa_{o}\left(g^{-1} k m\right)=\kappa_{o}\left(g^{-1} k\right) m$. Since $m \in M$ commutes with $A$ and $N$,

$$
g k m \in \kappa_{o}(g k) m \exp \left(H_{o}(g k)\right) N
$$

and so $k \mapsto H_{o}(g k)$ is $M$-invariant. It follows that $k \mapsto F(k) e^{-2 \rho\left(H_{o}(g k)\right)}$ is also $M$-invariant. The assertion follows by applying (31) to $F$ in place of $H$ and then rewriting the integrals over $K$ of the $M$-invariant functions as integrals over $K / M$ w.r.t. $v^{o}$ as before.

Now we investigate the relation between the different boundary measures introduced above. If $F \in C(K / M)$ and $x \in X$, then

$$
\begin{aligned}
\int_{K / M} F(k M) \mathrm{d} v^{x}(k M) & =\int_{K / M} F\left(\kappa_{o}\left(\tilde{T}_{x}(k M)\right)\right) \mathrm{d} v^{o}(k M) \\
& =\int_{K / M} F\left(\kappa_{o}\left(s_{o}(x) k\right) M\right) \mathrm{d} v^{o}(k M) \\
& =\int_{K / M} F(k M) e^{-2 \rho\left(H_{o}\left(s_{o}(x)^{-1} k\right)\right)} \mathrm{d} v^{o}(k M) \\
& =\int_{K / M} F(k M) e^{2 \rho\left(A_{o}(x, k M)\right)} \mathrm{d} v^{o}(k M)
\end{aligned}
$$

by Lemma 3 and then applying item (i) of Lemma 2 together with (14), since

$$
-H_{o}\left(s_{o}(x)^{-1} k\right)=A_{o}\left(k^{-1} s_{o}(x)\right)=A_{o}\left(s_{o}(x)[o], k M\right)=A_{o}(x, k M) .
$$

By expressing the integral of a function on $K / M$ with respect to either $v^{x}$ or $v^{y}$ as above and then using (22) in the form

$$
A_{o}(x, k M)=A_{o}(y, k M)+A_{y}(x, k M),
$$

the Radon-Nikodym derivative between the measures $v^{x}$ and $v^{y}$ is then

$$
\frac{\mathrm{d} v^{x}}{\mathrm{~d} v^{y}}(k M)=e^{2 \rho\left(A_{y}(x, k M)\right)} .
$$

Let $x \in X, g \in G$ and $F \in C(K / M)$. Using first (32) with $y=o$ and then (30) 


$$
\begin{aligned}
\int_{K / M} F\left(g^{-1}\langle k M\rangle\right) \mathrm{d} v^{x}(k M) & =\int_{K / M} F\left(g^{-1}\langle k M\rangle\right) e^{2 \rho\left(A_{o}(x, k M)\right)} \mathrm{d} v^{o}(k M) \\
& =\int_{K / M} F(k M) e^{2 \rho\left(A_{o}(x, g\langle k M\rangle)\right)} e^{-2 \rho\left(H_{o}(g k)\right)} \mathrm{d} v^{o}(k M) .
\end{aligned}
$$

Now observe that, by (20) and (21),

$$
\begin{aligned}
A_{o}(x, g\langle k M\rangle)-H_{o}(g k) & =A_{g^{-1}[o]}\left(g^{-1}[x], k M\right)+A_{o}\left(k^{-1} g^{-1}\right) \\
& =A_{g^{-1}[o]}\left(g^{-1}[x], k M\right)+A_{o}\left(g^{-1}[o], k M\right) \\
& =A_{o}\left(g^{-1}[x], k M\right),
\end{aligned}
$$

the latter equality being just (22) from Lemma 2. Hence, we obtain a sort of dual relation between the $G$-action on the boundary and that on the reference points of the boundary measures, namely

$$
\int_{K / M} F\left(g^{-1}\langle k M\rangle\right) \mathrm{d} v^{x}(k M)=\int_{K / M} F(k M) \mathrm{d} v^{g^{-1}[x]}(k M) .
$$

\subsubsection{Measures on $\Xi$}

Finally, in order to develop the theory in which we are interested, we need to introduce a $G$-invariant measure on $\Xi$. We denote by $\sigma$ the measure on $A$ with density $e^{2 \rho(\log a)}$ with respect to the Haar measure $\mathrm{d} a$. For every $x \in X$, we can endow $\Xi$ with the measure $\mathrm{d} \xi$ obtained as the pushforward of the measure $v^{x} \otimes \sigma$ on $K / M \times A$ by means of the map $\Psi_{x}$, i.e.

$$
\mathrm{d} \xi=\Psi_{x *}\left(v^{x} \otimes \sigma\right) .
$$

It turns out that $\mathrm{d} \xi$ is independent of the choice of $x \in X$. We denote by $L^{1}(\Xi)$ and $L^{2}(\Xi)$ the spaces of absolutely integrable functions and square-integrable functions with respect to the measure d $\xi$, respectively. By definition, for every $F \in L^{1}(\Xi)$

$$
\begin{aligned}
\int_{\Xi} F(\xi) \mathrm{d} \xi & =\int_{K / M \times A}\left(F \circ \Psi_{x}\right)(k M, a) \mathrm{d}\left(v^{x} \otimes \sigma\right)(k M, a) \\
& =\int_{K / M \times A}\left(F \circ \Psi_{x}\right)(k M, a) e^{2 \rho(\log a)} \mathrm{d} v^{x}(k M) \mathrm{d} a .
\end{aligned}
$$

It is easy to verify that $\mathrm{d} \xi$ is $G$-invariant. We point out that Helgason introduced this measure w.r.t. $o \in X$, see Lemma 3.1 in Chap. II in [17]. Since in our treatment it is important to change the reference point the expression above suits our needs.

The group $G$ acts on $L^{2}(\Xi)$ by the quasi-regular representation $\hat{\pi}: G \rightarrow$ $\mathcal{U}\left(L^{2}(\Xi)\right)$ defined by

$$
\hat{\pi}(g) F(\xi):=F\left(g^{-1} \cdot \xi\right), \quad F \in L^{2}(\Xi), g \in G .
$$

Equivalently, given $x \in X$, by (27) 


$$
(\hat{\pi}(g) F) \circ \Psi_{x}(k M, a)=F \circ \Psi_{g^{-1}[x]}\left(g^{-1}\langle k M\rangle, a\right),
$$

for every $(k M, a) \in K / M \times A$ and $g \in G$.

We need to introduce some more notation. We denote by $\Delta^{-\frac{1}{2}}$ the map on $K / M \times A$ defined by

$$
\Delta^{-\frac{1}{2}}(k M, a)=\mathrm{e}^{\rho(\log a)} .
$$

The reason for such notation resides in the fact that this function has the same expression of the inverse of the square root of the modular function of the $A N$ Iwasawa group, see (29).

Finally, for every $x \in X$, we introduce the space $L_{x}^{2}(K / M \times A)$ of square-integrable functions on $K / M \times A$ w.r.t. the measure $v^{x} \otimes \mathrm{d} a$. For every $F \in L^{2}(\Xi)$, we denote by $\Psi_{x}^{*} F$ the $\left(L^{2}(\Xi), L_{x}^{2}(K / M \times A)\right)$-pull-back of $F$ by $\Psi_{x}$, that is, we introduce the unitary operator $\Psi_{x}^{*}: L^{2}(\Xi) \rightarrow L_{x}^{2}(K / M \times A)$ given by

$$
\Psi_{x}^{*} F(k M, a)=\left(\Delta^{-\frac{1}{2}} \cdot\left(F \circ \Psi_{x}\right)\right)(k M, a)
$$

for almost every $(k M, a) \in K / M \times A$. In order to see that $\Psi_{x}^{*}$ is unitary, observe that for every $F \in L^{2}(\Xi)$ we have that

$$
\begin{aligned}
& \int_{K / M \times A}\left|\Psi_{x}^{*} F(k M, a)\right|^{2} \mathrm{~d} v^{x}(k M) \mathrm{d} a \\
& =\int_{K / M \times A}\left|\left(\Delta^{-\frac{1}{2}} \cdot\left(F \circ \Psi_{x}\right)\right)(k M, a)\right|^{2} \mathrm{~d} v^{x}(k M) \mathrm{d} a \\
& =\int_{K / M \times A}\left|\left(F \circ \Psi_{x}\right)(k M, a)\right|^{2} e^{2 \rho(\log a)} \mathrm{d} v^{x}(k M) \mathrm{d} a \\
& =\int_{\Xi}|F(\xi)|^{2} \mathrm{~d} \xi=\|F\|_{L^{2}(\Xi)}^{2},
\end{aligned}
$$

so that $\Psi_{x}^{*}$ is an isometry from $L^{2}(\Xi)$ into $L_{x}^{2}(K / M \times A)$. Surjectivity is also clear.

\subsection{The Helgason-Fourier Transform}

The Helgason-Fourier transform was defined by Helgason in analogy with the Fourier transform on Euclidean spaces in polar coordinates. We briefly recall its definition and its main features.

Definition 8 (\$1, Chap. III, [17]). The Helgason-Fourier transform of $f \in \mathcal{D}(X)$ is the function $\mathcal{H} f: K / M \times \mathfrak{a}^{*} \longrightarrow \mathbb{C}$ defined by

$$
\mathcal{H} f(k M, \lambda)=\int_{X} f(x) e^{(-i \lambda+\rho)\left(A_{o}(x, k M)\right)} \mathrm{d} x
$$


As the Euclidean Fourier transform, the Helgason-Fourier transform extends to a unitary operator on $L^{2}(X)$. The Plancherel measure involves the Harish-Chandra c function, a cornerstone in the analysis on symmetric spaces [13], [14]. It is a meromorphic function $\mathbf{c}: \mathfrak{a}_{c}^{*} \rightarrow \mathbb{C}$ defined on the complexified dual space $\mathfrak{a}_{c}^{*}$ for which various formulae are available (see e.g. [18]). It may thus be restricted to the real space $\mathfrak{a}^{*}$. As an example, in the case of the unit disk, if $\mathfrak{R}(i \lambda)>0$, then

$$
\mathbf{c}(\lambda)=\pi^{-1 / 2} \frac{\Gamma\left(\frac{1}{2} i \lambda\right)}{\Gamma\left(\frac{1}{2}(i \lambda+1)\right)},
$$

so that

$$
|\mathbf{c}(\lambda)|^{-2}=\frac{\pi \lambda}{2} \tanh \left(\frac{\pi \lambda}{2}\right) .
$$

We denote by $L_{o, \mathbf{c}}^{2}\left(K / M \times \mathfrak{a}^{*}\right)$ the space of the functions on $K / M \times \mathfrak{a}^{*}$ that are square-integrable w.r.t. the measure $w^{-1}|\mathbf{c}(\lambda)|^{-2} \mathrm{~d} v^{o} \mathrm{~d} \lambda$, where $w$ stands for the cardinality of the Weyl group $W$.

Proposition 7. For every $f_{1}, f_{2} \in \mathcal{D}(X)$

$$
\int_{x} f_{1}(x) \overline{f_{2}(x)} \mathrm{d} x=\int_{\mathfrak{a}^{*} \times K / M} \mathcal{H} f_{1}(k M, \lambda) \overline{\mathcal{H} f_{2}(k M, \lambda)} \mathrm{d} v^{o}(k M) \frac{\mathrm{d} \lambda}{w|\mathbf{c}(\lambda)|^{2}} .
$$

The rest of the paragraph is devoted to state the Plancherel theorem for the HelgasonFourier transform.

Property $\sharp$. We say that a function $F \in L_{o, \mathbf{c}}^{2}\left(K / M \times \mathfrak{a}^{*}\right)$ satisfies Property $\sharp$ if for every $x \in X$ the function

$$
\mathfrak{a}^{*} \ni \lambda \longmapsto \int_{K / M} e^{(\rho+i \lambda)\left(A_{o}(x, k M)\right)} F(k M, \lambda) \mathrm{d} v^{o}(k M)
$$

is $W$-invariant almost everywhere (see the comments after (5) for the $W$-action on $\left.\mathfrak{a}^{*}\right)$.

We denote by $L_{o, \mathbf{c}}^{2}\left(K / M \times \mathfrak{a}^{*}\right)^{\#}$ the space of functions $F$ in $L_{o, \mathbf{c}}^{2}\left(K / M \times \mathfrak{a}^{*}\right)$ satisfying Property $\sharp$. We observe that the integral in (36) is absolutely convergent for almost every $\lambda \in \mathfrak{a}^{*}$. By Fubini theorem, for every $F \in L_{o, \mathbf{c}}^{2}\left(K / M \times \mathfrak{a}^{*}\right)$ we have that

$$
\|F\|_{L_{o, \mathbf{c}}^{2}\left(K / M \times \mathbf{a}^{*}\right)}^{2}=\int_{\mathfrak{a}^{*}} \int_{K / M}|F(k M, \lambda)|^{2} \mathrm{~d} v^{o}(k M) \frac{\mathrm{d} \lambda}{w|\mathbf{c}(\lambda)|^{2}}<+\infty .
$$

Thus, the function $F(\cdot, \lambda)$ is in $L^{2}\left(K / M, v^{o}\right) \subseteq L^{1}\left(K / M, v^{o}\right)$ for almost every $\lambda \in \mathfrak{a}^{*}$ and, since $\rho\left(A_{o}(x, \cdot)\right)$ is bounded on $K / M$, the integrability properties of $F(\cdot, \lambda)$ continue to hold for the function $e^{(\rho+i \lambda)\left(A_{o}(x, \cdot)\right)} F(\cdot, \lambda)$.

Every function $F \in L_{o, \mathbf{c}}^{2}\left(K / M \times \mathfrak{a}^{*}\right)^{\#}$ is uniquely determined by its restriction on $K / M \times \mathfrak{a}_{+}^{*}$. Here $\mathfrak{a}_{+}^{*}$ denotes the positive Weyl chamber 


$$
\mathfrak{a}_{+}^{*}=\left\{\lambda \in \mathfrak{a}^{*}: A_{\lambda} \in \mathfrak{a}^{+}\right\},
$$

where $A_{\lambda}$ represents $\lambda$ via the Killing form, in the sense that $\lambda(H)=B\left(A_{\lambda}, H\right)$. If we suppose that $F, G \in L_{o, \mathbf{c}}^{2}\left(K / M \times \mathfrak{a}^{*}\right)^{\#}$ are such that $\left.F_{1}\right|_{K / M \times \mathbf{a}_{+}^{*}}=\left.F_{2}\right|_{K / M \times \mathfrak{a}_{+}^{*}}$, then

$$
\begin{aligned}
& \int_{K / M} e^{(\rho+i s \lambda)\left(A_{o}(x, k M)\right)}\left(F_{1}-F_{2}\right)(k M, s \lambda) \mathrm{d} v^{o}(k M) \\
& =\int_{K / M} e^{(\rho+i \lambda)\left(A_{o}(x, k M)\right)}\left(F_{1}-F_{2}\right)(k M, \lambda) \mathrm{d} v^{o}(k M)=0
\end{aligned}
$$

for a. e. $\lambda \in \mathfrak{a}_{+}^{*}$ and for every $s \in W$. Therefore, by Lemma 5.3 in Chap. II in [17], we can conclude that $F_{1}-F_{2}=0$ in $L_{o, c}^{2}\left(K / M \times \mathfrak{a}^{*}\right)$.

By the Paley-Wiener theorem for the Helgason Fourier transform (Theorem 5.1 in Chap. III in [17]), $\mathcal{H} f \in L_{o, \mathbf{c}}^{2}\left(K / M \times \mathfrak{a}^{*}\right)^{\sharp}$ for every $f \in \mathcal{D}(X)$, so that $\mathcal{H} f$ is uniquely determined by its restriction on $K / M \times \mathfrak{a}_{+}^{*}$. We denote by $L_{o, c}^{2}\left(K / M \times \mathfrak{a}_{+}^{*}\right)$ the space of the functions on $K / M \times \mathfrak{a}_{+}^{*}$ that are square-integrable w.r.t. the measure $|\mathbf{c}(\lambda)|^{-2} \mathrm{~d} v^{o} \mathrm{~d} \lambda$ and the Plancherel theorem for the Helgason-Fourier transform reads:

Theorem 8 (Theorem 1.5, Chap. III, [17]). The restricted Helgason-Fourier transform $\left.f \mapsto \mathcal{H} f\right|_{K / M \times \mathbf{a}_{+}^{*}}$ extends to a unitary operator $\mathscr{H}$ from $L^{2}(X)$ onto $L_{o, \mathbf{c}}^{2}\left(K / M \times \mathfrak{a}_{+}^{*}\right)$.

By the Plancherel formula (35), $\mathcal{H}$ is an isometry from $\mathcal{D}(X)$ into $L_{o, c}^{2}\left(K / M \times \mathfrak{a}^{*}\right)$. Furthermore, we show that, by Theorem $8, \mathcal{H}(\mathcal{D}(X))$ embeds densely in $L_{o, \mathbf{c}}^{2}(K / M \times$ $\left.\mathfrak{a}^{*}\right)^{\#}$. Let $F \in L_{o, c}^{2}\left(K / M \times \mathfrak{a}^{*}\right)^{\#}$ be such that $\langle F, \mathcal{H} f\rangle_{L_{o, \mathbf{c}}^{2}\left(K / M \times \mathfrak{a}^{*}\right)}=0$ for every $f \in \mathcal{D}(X)$. Then, by Fubini theorem we have that

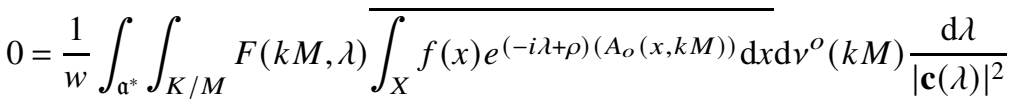

$$
\begin{aligned}
& =\frac{1}{w} \int_{\mathfrak{a}^{*}} \int_{X} \int_{K / M} F(k M, \lambda) e^{(i \lambda+\rho)\left(A_{o}(x, k M)\right)} \mathrm{d} v^{o}(k M) \overline{f(x)} \mathrm{d} x \frac{\mathrm{d} \lambda}{|\mathbf{c}(\lambda)|^{2}} \\
& =\int_{\mathfrak{a}_{+}^{*}} \int_{X} \int_{K / M} F(k M, \lambda) e^{(i \lambda+\rho)\left(A_{o}(x, k M)\right)} \mathrm{d} v^{o}(k M) \overline{f(x)} \mathrm{d} x \frac{\mathrm{d} \lambda}{|\mathbf{c}(\lambda)|^{2}} \\
& =\int_{\mathfrak{a}_{+}^{*}} \int_{K / M} F(k M, \lambda) \overline{\mathcal{H} f(k M, \lambda)} \mathrm{d} v^{o}(k M) \frac{\mathrm{d} \lambda}{|\mathbf{c}(\lambda)|^{2}},
\end{aligned}
$$

where we use that $F$ satisfies Property $\sharp$ and $|\mathbf{c}|^{2}$ is $W$-invariant. Hence, (37) yields

$$
\left\langle\left. F\right|_{K / M \times \mathfrak{a}_{+}^{*}},\left.\mathcal{H} f\right|_{K / M \times \mathfrak{a}_{+}^{*}}\right\rangle_{L_{o, \mathbf{c}}^{2}\left(K / M \times \mathfrak{a}_{+}^{*}\right)}=\left\langle\left. F\right|_{K / M \times \mathbf{a}_{+}^{*}}, \mathscr{H} f\right\rangle_{L_{o, \mathbf{c}}^{2}\left(K / M \times \mathfrak{a}_{+}^{*}\right)}=0,
$$

for every $f \in \mathcal{D}(X)$, and Theorem 8 implies that $F \equiv 0$ a.e. on $K / M \times \mathfrak{a}_{+}^{*}$. Therefore, $F=0$ in $L_{o, c}^{2}\left(K / M \times \mathfrak{a}^{*}\right)$ and $\mathcal{H}(\mathcal{D}(X))$ embeds densely in $L_{o, \mathbf{c}}^{2}\left(K / M \times \mathfrak{a}^{*}\right)^{\#}$.

We have the following equivalent version of Theorem 8 , that better suits our needs. 
Theorem 9. The Helgason-Fourier transform $\mathcal{H}$ extends to a unitary operator $\mathscr{H}$ from $L^{2}(X)$ onto $L_{o, \mathbf{c}}^{2}\left(K / M \times \mathfrak{a}^{*}\right)^{\#}$.

In what follows, we always consider $\mathscr{H}$ taking values in $L_{o, c}^{2}\left(K / M \times \mathfrak{a}^{*}\right)^{\sharp}$.

\subsection{The Horocyclic Radon Transform}

In what follows we introduce the horocyclic Radon transform, we study its range, and we investigate its intertwining properties with the quasi-regular representations $\pi$ and $\hat{\pi}$ of $G$.

Because horocycles admit (several) explicit parametrizations, we define the horocyclic Radon transform appealing directly to the basic parametrization, as clarified in the definition that follows.

Definition 9. The horocyclic Radon transform $\mathcal{R} f$ of a function $f \in \mathcal{D}(X)$ is the map $\mathcal{R} f: \Xi \rightarrow \mathbb{C}$ defined by

$$
\left(\mathcal{R} f \circ \Psi_{o}\right)(k M, a)=\int_{N} f(k a n[o]) \mathrm{d} n,
$$

for every $(k M, a) \in K / M \times A$.

If we change parametrization, and use equality (28), for any $x \in X$ we obtain the equivalent definition

$$
\begin{aligned}
\left(\mathcal{R} f \circ \Psi_{x}\right)(k M, a) & =\left(\mathcal{R} f \circ \Psi_{o}\right)\left(k M, a \exp \left(A_{o}(x, k M)\right)\right) \\
& =\int_{N} f\left(k a \exp \left(A_{o}(x, k M)\right) n[o]\right) \mathrm{d} n .
\end{aligned}
$$

Definition 10. Let $f \in \mathcal{D}(X)$. We denote by $\mathcal{A} f$ the map $\mathcal{A} f: K / M \times A \rightarrow \mathbb{C}$ defined by

$$
\mathcal{A} f(k M, a):=\Psi_{o}^{*}(\mathcal{R} f)(k M, a)=\left(\Delta^{-\frac{1}{2}} \cdot\left(\mathcal{R} f \circ \Psi_{o}\right)\right)(k M, a) .
$$

It is worth observing that if the function $f$ is $K$-bi-invariant, then $\mathcal{A} f$ coincides with the Abel transform of $f$ introduced by Helgason in Chap. III in [17].

We need to introduce the Fourier transform on the Abelian group A.

Definition 11 (\$4.2, Chap. 4, [8]). Let $s \in L^{1}(A)$. The Fourier transform $\mathcal{F} s$ of $s$ is defined on $\mathfrak{a}^{*}$ by

$$
\mathcal{F} s(\lambda)=\int_{A} s(a) e^{-i \lambda(\log a)} \mathrm{d} a .
$$

We now state a fundamental theorem in the $L^{2}$ theory of the Fourier transform. 
Theorem 10 (Theorem 4.26, Chap. 4, [8]). The Fourier transform $\mathcal{F}: L^{1} \cap$ $L^{2}(A) \rightarrow C\left(\mathfrak{a}^{*}\right)$ extends uniquely to a unitary operator from $L^{2}(A)$ onto $L^{2}\left(\mathfrak{a}^{*}\right)$. In particular,

$$
\|\mathcal{F} s\|_{L^{2}\left(\mathfrak{a}^{*}\right)}=\|s\|_{L^{2}(A)} .
$$

We denote by $R$ the regular representation of $A$ on $L^{2}(A)$, which is defined for every $s \in L^{2}(A)$ and for every $\alpha \in A$ by

$$
R_{\alpha} s(a)=s\left(\alpha^{-1} a\right), \quad a \in A .
$$

Furthermore, we denote by $M$ the representation of $A$ on $L^{2}\left(\mathfrak{a}^{*}\right)$ defined for every $r \in L^{2}\left(\mathfrak{a}^{*}\right)$ and for every $\alpha \in A$ by

$$
M_{\alpha} r(\lambda)=e^{-i \lambda(\log \alpha)} r(\lambda), \quad \lambda \in \mathfrak{a}^{*} .
$$

Proposition 8 (\$7.2, Chap. 5, [20]). The Fourier transform $\mathcal{F}: L^{2}(A) \rightarrow L^{2}\left(\mathfrak{a}^{*}\right)$ intertwines the regular representation $R$ with the representation $M$, i.e.

$$
\mathcal{F} R_{\alpha}=M_{\alpha} \mathcal{F}
$$

for every $\alpha \in A$.

We are now ready to recall the result which relates the Helgason-Fourier transform with the horocyclic Radon transform. We refer to Proposition 9 as the Fourier Slice Theorem for the horocyclic Radon transform in analogy with the polar Radon transform, see [19] as a classical reference. For the reader's convenience, we include the proof.

Proposition 9 (\$5, Chap. III, [17]). For every $f \in \mathcal{D}(X)$ and $k M \in K / M$, the function $a \mapsto \mathcal{A} f(k M, a)$ is in $L^{1}(A)$ and

$$
(I \otimes \mathcal{F}) \mathcal{A} f(k M, \lambda)=\mathcal{H} f(k M, \lambda),
$$

for almost every $\lambda \in \mathfrak{a}^{*}$.

Proof. If $f \in \mathcal{D}(X)$ and $k M \in K / M$, then by Proposition 6 and (20) 


$$
\begin{aligned}
\int_{A}|\mathcal{A} f(k M, a)| \mathrm{d} a & =\int_{A} e^{\rho(\log a)}\left|\mathcal{R} f \circ \Psi_{o}(k M, a)\right| \mathrm{d} a \\
& \leq \int_{A} \int_{N} e^{\rho(\log a)}|f(k a n[o])| \mathrm{d} n \mathrm{~d} a \\
& =\int_{A} \int_{N} \int_{K} e^{\rho(\log a)}\left|f\left(k a n k_{1}[o]\right)\right| \mathrm{d} k_{1} \mathrm{~d} n \mathrm{~d} a \\
& =\int_{G} e^{\rho\left(A_{o}(g)\right)}|f(k g[o])| \mathrm{d} g \\
& =\int_{G} e^{\rho\left(A_{o}\left(k^{-1} g\right)\right)}|f(g[o])| \mathrm{d} g \\
& =\int_{\operatorname{supp}(f)} e^{\rho\left(A_{o}(x, k M)\right)}|f(x)| \mathrm{d} x<+\infty
\end{aligned}
$$

Thus, $\mathcal{A} f(k M, \cdot)$ is in $L^{1}(A)$ and by similar steps it is easy to prove that

$$
(I \otimes \mathcal{F}) \mathcal{A} f(k M, \lambda)=\mathcal{H} f(k M, \lambda),
$$

for almost every $\lambda \in \mathfrak{a}^{*}$.

Let $f \in \mathcal{D}(X)$. By the Paley-Wiener theorem for the Helgason Fourier transform (Theorem 5.1 in Chap. III in [17]), $\mathcal{H} f$ is rapidly decreasing in the variable $\lambda \in \mathfrak{a}^{*}$ uniformly over $K / M$, that is for every $n \in \mathbb{N}$

$$
\|\mathcal{H} f\|_{n}:=\sup _{k M \in K / M, \lambda \in \mathfrak{a}^{*}}(1+|\lambda|)^{n}|\mathcal{H} f(k M, \lambda)|<+\infty .
$$

By Theorem 10 and Proposition 9, we have that

$$
\begin{aligned}
\int_{\Xi}|\mathcal{R} f(\xi)|^{2} \mathrm{~d} \xi & =\int_{K / M \times A}\left|\Psi_{o}^{*}(\mathcal{R} f)(k M, a)\right|^{2} \mathrm{~d} v^{o}(k M) \mathrm{d} a \\
& =\int_{K / M \times \mathfrak{a}^{*}}\left|(I \otimes \mathcal{F})\left(\Psi_{o}^{*}(\mathcal{R} f)\right)(k M, \lambda)\right|^{2} \mathrm{~d} v^{o}(k M) \mathrm{d} \lambda \\
& =\int_{K / M \times a^{*}}|\mathcal{H} f(k M, \lambda)|^{2} \mathrm{~d} v^{o}(k M) \mathrm{d} \lambda \\
& =\int_{K / M \times \mathbf{a}^{*}} \frac{(1+|\lambda|)^{2 n}|\mathcal{H} f(k M, \lambda)|^{2}}{(1+|\lambda|)^{2 n}} \mathrm{~d} v^{o}(k M) \mathrm{d} \lambda \\
& \leq\|\mathcal{H} f\|_{n}^{2} \int_{\mathfrak{a}^{*}} \frac{1}{(1+|\lambda|)^{2 n}} \mathrm{~d} \lambda<+\infty,
\end{aligned}
$$

for every $n>\operatorname{dim} A / 2$. Therefore, $\mathcal{R} f \in L^{2}(\Xi)$ for every $f \in \mathcal{D}(X)$.

The horocyclic Radon transform intertwines the regular representations $\pi$ and $\hat{\pi}$ of $G$.

Proposition 10. For every $g \in G$ and $f \in \mathcal{D}(X)$

$$
\mathcal{R}(\pi(g) f)=\hat{\pi}(g)(\mathcal{R} f) .
$$


Proof. Let $g \in G$ and $f \in \mathcal{D}(X)$. It is sufficient to show that $\mathcal{R}(\pi(g) f) \circ \Psi_{o}=$ $\hat{\pi}(g)(\mathcal{R} f) \circ \Psi_{o}$ on $K / M \times A$. Let $(k M, a) \in K / M \times A$. Then

$$
\begin{aligned}
\mathcal{R}(\pi(g) f) \circ \Psi_{o}(k M, a) & =\int_{N} \pi(g) f(k a n[o]) \mathrm{d} n \\
& =\int_{N} f\left(g^{-1} k a n[o]\right) \mathrm{d} n \\
& =\int_{N} f\left(\kappa_{o}\left(g^{-1} k\right) \exp \left(H_{o}\left(g^{-1} k\right)\right) a n[o]\right) \mathrm{d} n,
\end{aligned}
$$

where we used the decomposition $g^{-1} k \in \kappa_{o}\left(g^{-1} k\right) \exp \left(H_{o}\left(g^{-1} k\right)\right) N$ and the fact that $A$ normalizes $N$. Now, by (14), (20) and (22), we have

$$
H_{o}\left(g^{-1} k\right)=-A_{o}\left(k^{-1} g\right)=-A_{o}(g[o], k M)=A_{g[o]}(o, k M) .
$$

Finally, by $g^{-1}(k M)=\kappa_{o}\left(g^{-1} k\right) M$ and (38) we have that

$$
\begin{aligned}
\mathcal{R}(\pi(g) f) \circ \Psi_{o}(k M, a) & =\int_{N} f\left(\kappa_{o}\left(g^{-1} k\right) \exp \left(A_{g[o]}(o, k M)\right) \operatorname{an}[o]\right) \mathrm{d} n \\
& =\int_{N} f\left(\kappa_{o}\left(g^{-1} k\right) \exp \left(A_{o}\left(g^{-1}[o], g^{-1}\langle k M\rangle\right)\right) a n[o]\right) \mathrm{d} n \\
& =\mathcal{R} f \circ \Psi_{g^{-1}[o]}\left(g^{-1}\langle k M\rangle, a\right) \\
& =(\hat{\pi}(g) \mathcal{R} f) \circ \Psi_{o}(k M, a),
\end{aligned}
$$

where we used the action of $G$ on $\Xi$ given in (34).

We now introduce a closed subspace of $L^{2}(\Xi)$ which will play a crucial role because it is the range of the unitarization of the horocyclic Radon transform. By definition, for every $x \in X$ and every $F \in L^{2}(\Xi)$

$$
\|F\|_{L^{2}(\Xi)}^{2}=\int_{K / M} \int_{A}\left|\Psi_{x}^{*} F(k M, a)\right|^{2} \mathrm{~d} a \mathrm{~d} v^{x}(k M)<+\infty .
$$

So that, the function $\Psi_{x}^{*} F(k M, \cdot)$ is in $L^{2}(A)$ for almost every $k M \in K / M$. Then, by Plancherel formula and Fubini theorem

$$
\begin{aligned}
\|F\|_{L^{2}(\Xi)}^{2} & =\int_{K / M \times A}\left|\Psi_{x}^{*} F(k M, a)\right|^{2} \mathrm{~d} v^{x}(k M) \mathrm{d} a \\
& =\int_{K / M \times \mathfrak{a}^{*}}\left|(I \otimes \mathcal{F}) \Psi_{x}^{*} F(k M, \lambda)\right|^{2} \mathrm{~d} v^{x}(k M) \mathrm{d} \lambda \\
& =\int_{\mathfrak{a}^{*}} \int_{K / M}\left|(I \otimes \mathcal{F}) \Psi_{x}^{*} F(k M, \lambda)\right|^{2} \mathrm{~d} v^{x}(k M) \mathrm{d} \lambda<+\infty .
\end{aligned}
$$

So that, for almost every $\lambda \in \mathfrak{a}^{*}$ the function $(I \otimes \mathcal{F}) \Psi_{x}^{*} F(\cdot, \lambda)$ is in $L^{2}\left(K / M, v^{x}\right) \subseteq$ $L^{1}\left(K / M, v^{x}\right)$ and 


$$
\begin{aligned}
& \left|\int_{K / M}(I \otimes \mathcal{F}) \Psi_{x}^{*} F(k M, \lambda) \mathrm{d} v^{x}(k M)\right| \\
& \leq \int_{K / M}\left|(I \otimes \mathcal{F}) \Psi_{x}^{*} F(k M, \lambda)\right| \mathrm{d} v^{x}(k M)<+\infty .
\end{aligned}
$$

Property $b$. We say that a function $F \in L^{2}(\Xi)$ satisfies Property $b$ if for every $x \in X$ the function

$$
\mathfrak{a}^{*} \ni \lambda \longmapsto \int_{K / M}(I \otimes \mathcal{F}) \Psi_{x}^{*} F(k M, \lambda) \mathrm{d} v^{x}(k M)
$$

is $W$-invariant almost everywhere.

We denote by $L_{b}^{2}(\Xi)$ the space of functions $F \in L^{2}(\Xi)$ satisfying Property b. Notice that by the considerations above, the integral appearing in Property $b$ is finite for almost every $\lambda \in \mathfrak{a}^{*}$. Our main results in Sect. 5 are based on the characterization of $L_{b}^{2}(\Xi)$ given in Proposition 11 below. We denote by $L_{o}^{2}\left(K / M \times \mathfrak{a}^{*}\right)$ the space of square-integrable functions on $K / M \times \mathfrak{a}^{*}$ w.r.t. the measure $v^{o} \otimes \mathrm{d} \lambda$.

Proposition 11. The operator $\Phi_{o}$ defined on $F \in L^{2}(\Xi)$ by

$$
\Phi_{o} F(k M, \lambda)=(I \otimes \mathcal{F}) \Psi_{o}^{*} F(k M, \lambda), \quad \text { a.e. }(k M, \lambda) \in K / M \times \mathfrak{a}^{*},
$$

is an isometry from $L^{2}(\Xi)$ into $L_{o}^{2}\left(K / M \times \mathfrak{a}^{*}\right)$. Furthermore, a function $F$ belongs to $L_{b}^{2}(\Xi)$ if and only if $\Phi_{o} F$ satisfies Property $\sharp$.

Proof. By Parseval identity, for every $F \in L^{2}(\Xi)$ we have that

$$
\begin{aligned}
& \int_{K / M \times \mathfrak{a}^{*}}\left|\Phi_{o} F(k M, \lambda)\right|^{2} \mathrm{~d} v^{o}(k M) \mathrm{d} \lambda \\
& =\int_{K / M} \int_{\mathfrak{a}^{*}}\left|(I \otimes \mathcal{F}) \Psi_{o}^{*} F(k M, \lambda)\right|^{2} \mathrm{~d} \lambda \mathrm{d} v^{o}(k M) \\
& =\int_{K / M \times A}\left|\Psi_{o}^{*} F(k M, a)\right|^{2} \mathrm{~d} v^{o}(k M) \mathrm{d} a=\|F\|_{L^{2}(\Xi)}^{2},
\end{aligned}
$$

so that $\Phi_{o}$ is an isometry from $L^{2}(\Xi)$ into $L_{o}^{2}\left(K / M \times \mathfrak{a}^{*}\right)$. Now, let $F \in L^{2}(\Xi)$. By equation (28) and by the definition of the regular representation $R$ of $A$, for almost every $k M \in K / M$ and $\lambda \in \mathfrak{a}^{*}$ we have that

$$
\begin{aligned}
& \Phi_{o} F(k M, \lambda)=(I \otimes \mathcal{F}) \Psi_{o}^{*} F(k M, \lambda)=(I \otimes \mathcal{F})\left(\Delta^{-\frac{1}{2}} \cdot\left(F \circ \Psi_{o}\right)\right)(k M, \lambda)
\end{aligned}
$$

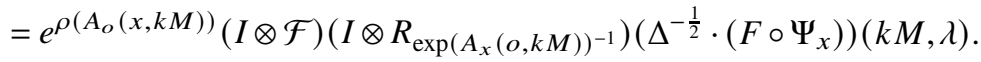

Therefore, by Proposition 8 we obtain 


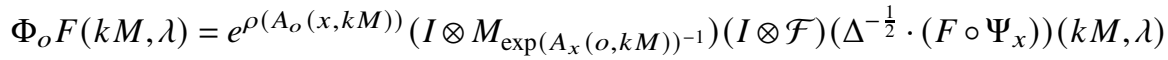

$$
\begin{aligned}
& =e^{(\rho-i \lambda)\left(A_{o}(x, k M)\right)}(I \otimes \mathcal{F})\left(\Delta^{-\frac{1}{2}} \cdot\left(F \circ \Psi_{x}\right)\right)(k M, \lambda) \\
& =e^{(\rho-i \lambda)\left(A_{o}(x, k M)\right)}(I \otimes \mathcal{F}) \Psi_{x}^{*} F(k M, \lambda) .
\end{aligned}
$$

Now, for every $x \in X$ and for almost every $\lambda \in \mathfrak{a}^{*}$, (40) yields

$$
\begin{aligned}
& \int_{K / M} e^{(\rho+i \lambda)\left(A_{o}(x, k M)\right)} \Phi_{o} F(k M, \lambda) \mathrm{d} v^{o}(k M) \\
& =\int_{K / M} e^{(\rho+i \lambda)\left(A_{o}(x, k M)\right)} e^{(\rho-i \lambda)\left(A_{o}(x, k M)\right)}(I \otimes \mathcal{F}) \Psi_{x}^{*} F(k M, \lambda) \mathrm{d} v^{o}(k M) \\
& =\int_{K / M}(I \otimes \mathcal{F}) \Psi_{x}^{*} F(k M, \lambda) e^{2 \rho\left(A_{o}(x, k M)\right)} \mathrm{d} v^{o}(k M) \\
& =\int_{K / M}(I \otimes \mathcal{F}) \Psi_{x}^{*} F(k M, \lambda) \mathrm{d} v^{x}(k M) .
\end{aligned}
$$

Equality (41) allows us to conclude that $F$ satisfies Property b if and only if $\Phi_{o} F$ satisfies Property $\sharp$ and this concludes our proof.

Corollary 1. For every $f \in \mathcal{D}(X)$,

$$
\Phi_{o}(\mathcal{R} f)=\mathcal{H} f
$$

in $L_{o}^{2}\left(K / M \times \mathfrak{a}^{*}\right)$ and $\mathcal{R} f \in L_{b}^{2}(\Xi)$.

Proof. The proof follows immediately by Proposition 9 and the fact that the Helgason-Fourier transform satisfies Property $\sharp$.

Some comments are in order. Proposition 11 with Corollary 1 shows the link between the range of the Radon transform with the range of the Helgason-Fourier transform, which will play a crucial role in our main result. The range $\mathcal{R}(\mathcal{D}(X))$ has already been completely characterized in Chap. IV in [17]. As it will be made clear in the next section, Property $b$ better suits our needs.

\section{Unitarization and Intertwining}

In order to obtain the unitarization for the horocyclic Radon transform that we are after, we need some technicalities. Figure 4 below might help the reader to keep track of all the spaces and operators involved in our construction.

We put

$$
\mathcal{D}_{o}=\left\{\varphi \in L_{o}^{2}(K / M \times A):(I \otimes \mathcal{F}) \varphi \in L_{o, c}^{2}\left(K / M \times \mathfrak{a}^{*}\right)\right\}
$$

and we define the operator $\mathcal{J}_{o}: \mathcal{D}_{o} \subseteq L_{o}^{2}(K / M \times A) \rightarrow L_{o}^{2}(K / M \times A)$ as the Fourier multiplier 


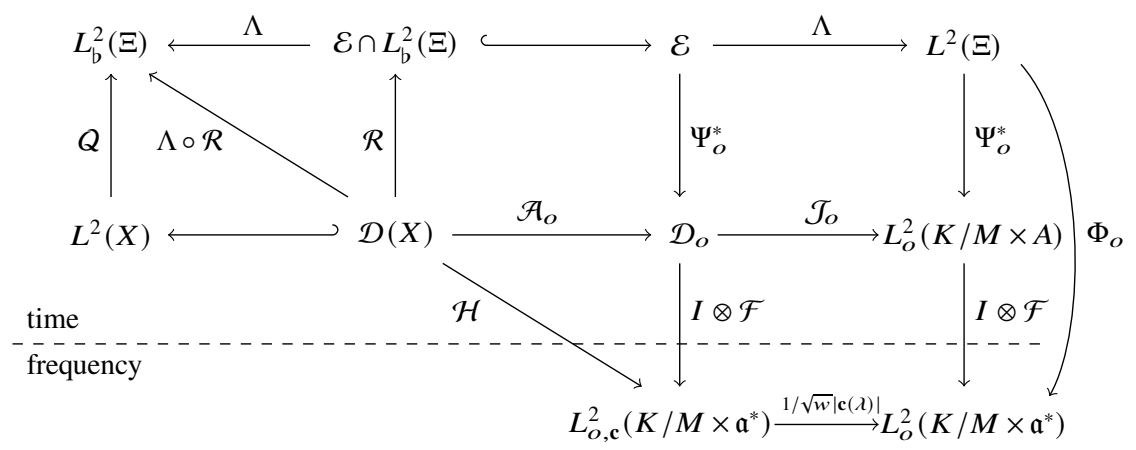

Fig. 4: Spaces and operators that come into play in our construction.

$(I \otimes \mathcal{F})\left(\mathcal{J}_{\diamond} \varphi\right)(k M, \lambda)=\frac{1}{\sqrt{w}|\mathbf{c}(\lambda)|}(I \otimes \mathcal{F}) \varphi(k M, \lambda), \quad$ a.e. $(k M, \lambda) \in K / M \times \mathfrak{a}^{*}$

We define the set of functions

$$
\mathcal{E}=\left\{F \in L^{2}(\Xi): \Phi_{o} F \in L_{o, c}^{2}\left(K / M \times \mathfrak{a}^{*}\right)\right\}
$$

and we consider the operator $\Lambda: \mathcal{E} \subseteq L^{2}(\Xi) \rightarrow L^{2}(\Xi)$ given by

$$
\Lambda F=\Psi_{o}^{*-1} \mathcal{J}_{o} \Psi_{o}^{*} F
$$

As a direct consequence of the definition of $\Lambda$ and $\mathcal{J}_{o}$, for every $F \in \mathcal{E}$ and for almost every $(k M, \lambda) \in K / M \times \mathfrak{a}^{*}$ we have (see the rightmost block in Fig. 4)

$$
\begin{aligned}
\Phi_{o}(\Lambda F)(k M, \lambda) & =(I \otimes \mathcal{F})\left(\mathcal{J}_{o} \Psi_{o}^{*} F\right)(k M, \lambda) \\
& =\frac{1}{\sqrt{w}|\mathbf{c}(\lambda)|}(I \otimes \mathcal{F})\left(\Psi_{o}^{*} F\right)(k M, \lambda) \\
& =\frac{1}{\sqrt{w}|\mathbf{c}(\lambda)|} \Phi_{o} F(k M, \lambda) .
\end{aligned}
$$

The operator $\Lambda$ intertwines the regular representation $\hat{\pi}$ as shown by the next proposition.

Proposition 12. The subspace $\mathcal{E}$ is $\hat{\pi}$-invariant and for all $F \in \mathcal{E}$ and $g \in G$

$$
\hat{\pi}(g) \Lambda F=\Lambda \hat{\pi}(g) F .
$$

Proof. We consider $F \in \mathcal{E}, g \in G$ and we prove that $\hat{\pi}(g) F \in \mathcal{E}$. By (34)

$$
\hat{\pi}(g) F \circ \Psi_{o}(k M, a)=F \circ \Psi_{g^{-1}[o]}\left(g^{-1}\langle k M\rangle, a\right)
$$

for almost every $(k M, a) \in K / M \times A$. Therefore, we have 


$$
\Psi_{o}^{*}(\hat{\pi}(g) F)(k M, a)=\Psi_{g^{-1}[o]}^{*} F\left(g^{-1}\langle k M\rangle, a\right)
$$

and consequently by equation (40)

$$
\begin{aligned}
\Phi_{o}(\hat{\pi}(g) F)(k M, \lambda) & =\left(I \otimes \mathcal{F}_{A}\right)\left(\Psi_{g^{-1}[o]}^{*} F\right)\left(g^{-1}\langle k M\rangle, \lambda\right) \\
& =e^{(\rho-i \lambda)\left(A_{g^{-1}[o]}\left(o, g^{-1}\langle k M\rangle\right)\right)} \Phi_{o}(F)\left(g^{-1}\langle k M\rangle, \lambda\right)
\end{aligned}
$$

for almost every $(k M, \lambda) \in K / M \times \mathfrak{a}^{*}$. By equations (44), (33) and (32)

$$
\begin{aligned}
& \int_{K / M \times \mathfrak{a}^{*}}\left|\Phi_{o}(\hat{\pi}(g) F)(k M, \lambda)\right|^{2} \frac{\mathrm{d} v^{o}(k M) \mathrm{d} \lambda}{w|\mathbf{c}(\lambda)|^{2}} \\
& =\int_{\mathfrak{a}^{*}} \int_{K / M}\left|\Phi_{o}(F)\left(g^{-1}\langle k M\rangle, \lambda\right)\right|^{2} e^{2 \rho\left(A_{g^{-1}[o]}\left(o, g^{-1}\langle k M\rangle\right)\right)} \frac{\mathrm{d} v^{o}(k M) \mathrm{d} \lambda}{w|\mathbf{c}(\lambda)|^{2}} \\
& =\int_{K / M \times \mathfrak{a}^{*}}\left|\Phi_{o} F(k M, \lambda)\right|^{2} e^{2 \rho\left(A_{g^{-1}[o]}(o, k M)\right)} \frac{\mathrm{d} v^{g^{-1}[o]}(k M) \mathrm{d} \lambda}{w|\mathbf{c}(\lambda)|^{2}} \\
& =\int_{K / M \times \mathfrak{a}^{*}}\left|\Phi_{o} F(k M, \lambda)\right|^{2} \frac{\mathrm{d} v^{o}(k M) \mathrm{d} \lambda}{w|\mathbf{c}(\lambda)|^{2}}<+\infty
\end{aligned}
$$

and we conclude that $\hat{\pi}(g) F \in \mathcal{E}$. We next prove the intertwining property (43). We have already observed that, by Proposition 11, it is enough to prove that

$$
\Phi_{o}(\hat{\pi}(g) \Lambda F)=\Phi_{o}(\Lambda \hat{\pi}(g) F)
$$

for every $g \in G$ and $F \in \mathcal{E}$. By equations (44) and (42), for almost every $(k M, \lambda) \in$ $K / M \times \mathfrak{a}^{*}$, we have the chain of equalities

$$
\begin{aligned}
\Phi_{o}(\hat{\pi}(g) \Lambda F)(k M, \lambda) & =e^{(\rho-i \lambda)\left(A_{g^{-1}[o]}\left(o, g^{-1}\langle k M\rangle\right)\right)} \Phi_{o}(\Lambda F)\left(g^{-1}\langle k M\rangle, \lambda\right) \\
& =\frac{1}{\sqrt{w}|\mathbf{c}(\lambda)|} e^{(\rho-i \lambda)\left(A_{g^{-1}[o]}\left(o, g^{-1}\langle k M\rangle\right)\right)} \Phi_{o}(F)\left(g^{-1}\langle k M\rangle, \lambda\right) \\
& =\frac{1}{\sqrt{w}|\mathbf{c}(\lambda)|} \Phi_{o}(\hat{\pi}(g) F)(k M, \lambda)=\Phi_{o}(\Lambda \hat{\pi}(g) F)(k M, \lambda),
\end{aligned}
$$

which proves the intertwining relation.

The next result follows directly by Proposition 11 and equation (42).

Corollary 2. For every $F \in \mathcal{E}, \Lambda F \in L_{b}^{2}(\Xi)$ if and only if $F \in L_{b}^{2}(\Xi)$.

Proof. By Proposition $11, \Lambda F \in L_{b}^{2}(\Xi)$ if and only if $\Phi_{o}(\Lambda F)$ satisfies Property $\sharp$. By (42) and since $\lambda \mapsto|\mathbf{c}(\lambda)|$ is $W$-invariant, $\Phi_{o}(\Lambda F)$ satisfies Property $\sharp$ if and only if $\Phi_{o}(F)$ satisfies Property $\sharp$, which is equivalent to $F \in L_{b}^{2}(\Xi)$. This concludes the proof.

We are now in a position to prove our main result.

Theorem 11. The composite operator $\Lambda \mathcal{R}$ extends to a unitary operator 


$$
Q: L^{2}(X) \longrightarrow L_{b}^{2}(\Xi)
$$

which intertwines the representations $\pi$ and $\hat{\pi}$, i.e.

$$
\hat{\pi}(g) Q=Q \pi(g), \quad g \in G .
$$

Theorem 11 implies that $\pi$ and the restriction $\left.\hat{\pi}\right|_{L_{b}^{2}(\Xi)}$ of $\hat{\pi}$ to $L_{b}^{2}(\Xi)$ are unitarily equivalent representations. Moreover, $\left.\hat{\pi}\right|_{L_{b}^{2}(\Xi)}$ (and then $\left.\hat{\pi}\right)$ is not irreducible, too.

Proof. We first show that $\Lambda \mathcal{R}$ extends to a unitary operator $Q$ from $L^{2}(X)$ onto $L^{2}(\Xi)$. It might be useful to keep in mind see the leftmost block in Fig. 4. Let $f \in \mathcal{D}(X)$, by the Fourier Slice Theorem (39), the Plancherel formula and the definition of $\mathcal{J}_{o}$ and $\Lambda$, we have that

$$
\begin{aligned}
\|f\|_{L^{2}(X)}^{2} & =\|\mathcal{H} f\|_{L_{o, c}^{2}\left(K / M \times \mathfrak{a}^{*}\right)^{\sharp}}^{2} \\
& =\left\|(I \otimes \mathcal{F})\left(\Psi_{o}^{*}(\mathcal{R} f)\right)\right\|_{L_{o, c}^{2}\left(K / M \times \mathfrak{a}^{*}\right)^{\sharp}}^{2} \\
& =\int_{K / M \times \mathfrak{a}^{*}}\left|(I \otimes \mathcal{F})\left(\mathcal{J}_{o} \Psi_{o}^{*}(\mathcal{R} f)\right)(k M, \lambda)\right|^{2} \mathrm{~d} v^{o}(k M) \mathrm{d} \lambda \\
& =\int_{K / M \times \mathfrak{a}^{*}}\left|(I \otimes \mathcal{F})\left(\Psi_{o}^{*}(\Lambda \mathcal{R} f)\right)(k M, \lambda)\right|^{2} \mathrm{~d} v^{o}(k M) \mathrm{d} \lambda \\
& =\int_{K / M \times A}\left|\Psi_{o}^{*}(\Lambda \mathcal{R} f)(k M, a)\right|^{2} \mathrm{~d} v^{o}(k M) \mathrm{d} a \\
& =\|\Lambda \mathcal{R} f\|_{L^{2}(\Xi)}^{2} .
\end{aligned}
$$

Hence, $\Lambda \mathcal{R}$ is an isometric operator from $\mathcal{D}(X)$ into $L^{2}(\Xi)$. Since $\mathcal{D}(X)$ is dense in $L^{2}(X), \Lambda \mathcal{R}$ extends to a unique isometry from $L^{2}(X)$ onto the closure of $\operatorname{Ran}(\Lambda \mathcal{R})$ in $L^{2}(\Xi)$. We must show that $\Lambda \mathcal{R}$ has dense image in $L_{b}^{2}(\Xi)$. The inclusion $\operatorname{Ran}(\Lambda \mathcal{R}) \subseteq$ $L_{b}^{2}(\Xi)$ follows immediately from Corollary 1 and Corollary 2. Let $F \in L_{b}^{2}(\Xi)$ be such that $\langle F, \Lambda \mathcal{R} f\rangle_{L^{2}(\Xi)}=0$ for every $\mathcal{D}(X)$. By the Plancherel formula and the Fourier Slice Theorem (39) we have that

$$
\begin{aligned}
0 & =\langle F, \Lambda \mathcal{R} f\rangle_{L^{2}(\Xi)} \\
& =\int_{K / M \times A}\left(F \circ \Psi_{o}\right)(k M, a) \overline{\left(\Lambda \mathcal{R} f \circ \Psi_{o}\right)(k M, a)} e^{2 \rho(\log a)} \mathrm{d} v^{o}(k M) \mathrm{d} a \\
& =\int_{K / M \times A}\left(\Psi_{o}^{*} F\right)(k M, a) \overline{\left(\mathcal{J}_{o} \Psi_{o}^{*}(\mathcal{R} f)(k M, a)\right.} \mathrm{d} v^{o}(k M) \mathrm{d} a \\
& =\int_{K / M \times \mathfrak{a}^{*}} \Phi_{o}(F)(k M, \lambda) \overline{(I \otimes \mathcal{F})\left(\mathcal{J}_{o} \Psi_{o}^{*}(\mathcal{R} f)\right)(k M, \lambda)} \mathrm{d} v^{o}(k M) \mathrm{d} \lambda \\
& =\int_{K / M \times \mathfrak{a}^{*}} \Phi_{o}(F)(k M, \lambda) \overline{(I \otimes \mathcal{F})\left(\Psi_{o}^{*}(\mathcal{R} f)\right)(k M, \lambda)} \frac{\mathrm{d} v^{o}(k M) \mathrm{d} \lambda}{\sqrt{w}|\mathbf{c}(\lambda)|} \\
& =\int_{K / M \times \mathfrak{a}^{*}} \sqrt{w}|\mathbf{c}(\lambda)| \Phi_{o}(F)(k M, \lambda) \overline{\mathcal{H}_{o} f(k M, \lambda)} \frac{\mathrm{d} v^{o}(k M) \mathrm{d} \lambda}{w|\mathbf{c}(\lambda)|^{2}} .
\end{aligned}
$$


For simplicity, we denote by $\Theta F$ the function on $K / M \times \mathfrak{a}^{*}$ defined as

$$
\Theta F(k M, \lambda)=\sqrt{w}|\mathbf{c}(\lambda)| \Phi_{o}(F)(k M, \lambda), \quad \text { a.e. }(k M, \lambda) \in K / M \times \mathfrak{a}^{*} .
$$

Hence we have proved that $\langle\Theta F, \mathcal{H} f\rangle=0$ for every $f \in \mathcal{D}(X)$. The next two facts follow immediately by Proposition 11. Since $\Phi_{o}$ is an isometry from $L^{2}(\Xi)$ into $L_{o}^{2}\left(K / M \times \mathfrak{a}^{*}\right)$, the function $\Theta F$ belongs to $L_{o, c}^{2}\left(K / M \times \mathfrak{a}^{*}\right)$. Further, since $F \in$ $L_{b}^{2}(\Xi)$ and since $\lambda \mapsto|\mathbf{c}(\lambda)|$ is $W$-invariant, then $\Theta F \in L_{o, c}^{2}\left(K / M \times \mathfrak{a}^{*}\right)^{\sharp}$. By Theorem $8, \mathcal{H}(\mathcal{D}(X))$ is dense in $L_{o, c}^{2}\left(K / M \times \mathfrak{a}^{*}\right)^{\#}$. Hence, $\Theta F=0$ in $L_{o, c}^{2}\left(K / M \times \mathfrak{a}^{*}\right)^{\#}$ and then $\Phi_{o}(F)=0$ in $L_{o}^{2}\left(K / M \times \mathfrak{a}^{*}\right)$. Since $\Phi_{o}$ is an isometry from $L^{2}(\Xi)$ into $L_{o}^{2}\left(K / M \times \mathfrak{a}^{*}\right)$, then $F=0$ in $L^{2}(\Xi)$. Therefore, $\overline{\operatorname{Ran}(\Lambda \mathcal{R})}=L_{\mathrm{b}}^{2}(\Xi)$ and $\Lambda \mathcal{R}$ extends uniquely to a surjective isometry

$$
Q: L^{2}(X) \longrightarrow L_{b}^{2}(\Xi)
$$

Observe that $Q f=\Lambda \mathcal{R} f$ for every $f \in \mathcal{D}(X)$. The intertwining property (45) follows immediately from Proposition 10 and Proposition 12.

\section{References}

1. G. S. Alberti, F. Bartolucci, F. De Mari, and E. De Vito. Unitarization and inversion formulae for the Radon transform between dual pairs. SIAM Journal on Mathematical Analysis, 51(6):43564381, 2019.

2. Giovanni Alberti, Francesca Bartolucci, Filippo De Mari, and Ernesto De Vito. Radon transform: Dual pairs and irreducible representations, 022020.

3. J.-P. Antoine and R. Murenzi. Two-dimensional directional wavelets and the scale-angle representation. Signal processing, 52(3):259-281, 1996.

4. F. Bartolucci, F. De Mari, E. De Vito, and F. Odone. The Radon transform intertwines wavelets and shearlets. Applied and Computational Harmonic Analysis, 47(3):822-847, 2019.

5. F. Bartolucci, F. De Mari, and M. Monti. Unitarization of the Radon transform on homogeneous trees. Submitted, arXiv:2002.06696v2, 2020.

6. S. Dahlke, G. Steidl, and G. Teschke. The continuous shearlet transform in arbitrary space dimensions. Journal of Fourier Analysis and Applications, 16(3):340-364, 2010.

7. M. Duflo and C. C. Moore. On the regular representation of a nonunimodular locally compact group. J. Functional Analysis, 21(2):209-243, 1976.

8. G. B. Folland. A course in abstract harmonic analysis. Textbooks in Mathematics. CRC Press, Boca Raton, FL, second edition, 2016.

9. H. Führ. Continuous wavelet transforms with abelian dilation groups. J. Math. Phys., 39(8):3974-3986, 1998.

10. H. Führ and R. R. Tousi. Simplified vanishing moment criteria for wavelets over general dilation groups, with applications to abelian and shearlet dilation groups. Appl. Comput. Harmon. Anal., 43(3):449-481, 2017.

11. H. Furstenberg. A Poisson formula for semi-simple Lie groups. Annals of Mathematics, pages 335-386, 1963.

12. I M Gel'fand. Integral geometry and its relation to the theory of group representations. 15(2):143-151, apr 1960.

13. Harish-Chandra. Spherical functions on a semisimple Lie group, I. American Journal of Mathematics, 80(2):241-310, 1958. 
14. Harish-Chandra. Spherical functions on a semisimple Lie group II. American Journal of Mathematics, 80(3):553-613, 1958.

15. S. Helgason. Differential geometry, Lie groups, and symmetric spaces, volume 80. Academic press, 1979.

16. S. Helgason. Groups \& geometric analysis: Radon transforms, invariant differential operators and spherical functions, volume 1. Academic press, 1984.

17. S. Helgason. Geometric analysis on symmetric spaces. Providence, RI, 1994.

18. S. Helgason. Harish-Chandra's c-function. A mathematical jewel. In Noncompact Lie groups and some of their applications, pages 55-67. Springer, 1994.

19. S. Helgason. The Radon transform, volume 5 of Progress in Mathematics. Birkhäuser Boston, Inc., Boston, MA, second edition, 1999.

20. M. Holschneider. Wavelets. Oxford Mathematical Monographs. The Clarendon Press, Oxford University Press, New York, 1995. An analysis tool, Oxford Science Publications.

21. A. Iozzi. Symmetric spaces. 2014.

22. A. W. Knapp. Representation theory of semisimple groups: an overview based on examples, volume 36. Princeton university press, 2001.

23. D. Labate, W.-Q. Lim, G. Kutyniok, and G. Weiss. Sparse multidimensional representation using shearlets. In Optics \& Photonics 2005, pages 59140U-59140U. International Society for Optics and Photonics, 2005.

24. F. Rouviere. Geodesic Radon transforms on symmetric spaces. 012004.

25. P. Sardar. Geometry of the symmetric space $\operatorname{SL}(n, \mathbb{R}) / \mathrm{SO}(\mathrm{n}, \mathbb{R})$, geometry, groups and dynamics $(G G D)$. https://www.youtube.com/watch?v=SnfYvKJIxrg\&t=2341s, 2017.

26. V. S. Varadarajan. Geometry of quantum theory. Springer-Verlag, New York, 2nd edition, 1985.

27. F. W. Warner. Foundations of differentiable manifolds and Lie groups, volume 94 of Graduate Texts in Mathematics. Springer-Verlag, New York-Berlin, 1983. Corrected reprint of the 1971 edition.

28. J. A. Wolf. Harmonic analysis on commutative spaces, volume 142 of Mathematical Surveys and Monographs. American Mathematical Society, Providence, RI, 2007. 